\title{
Chaotic oscillations in a nearly inviscid, axisymmetric capillary bridge at 2:1 parametric resonance
}

\author{
Francisco J. Mancebo, José A. Nicolás, and José M. Vegaa) \\ E.T.S.I. Aeronáuticos, Universidad Politécnica, Madrid. Spain
}

\begin{abstract}
We consider the 2:I internal resonances (such that $\Omega_{1}>0$ and $\Omega_{2} \simeq 2 \Omega_{1}$ are natural frequencies) that appear in a nearly inviscid. axisymmetric capillary bridge when the slenderness $\Lambda$ is such that $0<\Lambda<\pi$ (to avoid the Rayleigh instability) and only the first eight capillary modes are considered. A normal form is derived that gives the slow evolution (in the viscous time scale) of the complex amplitudes of the eigenmodes associated with $\Omega_{1}$ and $\Omega_{2}$, and consists of two complex ODEs that are balances of terms accounting for inertia, damping, detuning from resonance. quadratic nonlinearity. and forcing. In order to obtain quantitatively good results. a two-term approximation is used for the damping rate. The coefficients of quadratic terms are seen to be nonzero if and only if the eigenmode associated with $\Omega_{2}$ is even. In that case the quadratic normal form possesses steady states (which correspond to mono- or bichromatic oscillations of the liquid bridge) and more complex periodic or chaotic attractors (corresponding to periodically or chaotically modulated oscillations). For illustration, several bifurcation diagrams are analyzed in some detail for an intemal resonance that appears at $\Lambda \simeq 2.23$ and involves the fifth and eighth eigenmodes. If, instead, the eigenmode associated with $\Omega_{2}$ is odd, and only one of the eigenmodes associated with $\Omega_{1}$ and $\Omega_{2}$ is directly excited, then quadratic terms are absent in the normal form and the associated dynamics is seen to be fairly simple.
\end{abstract}

\section{INTRODUCTION}

Capillary bridges have received fairly great attention in the literature in the last 20 years due to their intrinsic interest as simple configurations exhibiting interesting spatiotemporal behavior. In addition, these liquid configurations are of interest in some industrial processes (crystal growth from the melt ${ }^{1-3}$ ) and natural phenomena (flow through porous media, ${ }^{4,5}$ particulates agglomeration ${ }^{6}$ ). They are also useful for the experimental measurement of viscosity and surface tension. $^{7}$

Theoretical analyses of mechanical oscillations of liquid bridges have been mainly based on one-dimensional approximations $^{8-11}$ (which exhibit several limitations ${ }^{12}$ ) and/or concerned with the linear approximation. ${ }^{7,13-17}$ Among the few exceptions to this rule, there are two recent papers by Chen and Tsamopoulos ${ }^{18}$ and Nicolás and Vega, ${ }^{19}$ who analyzed fully nonlinear oscillations by direct numerical simulation and weakly nonlinear oscillations by asymptotic methods, respectively. These two papers may be seen as complementary of each other. The results in Ref. 18 apply only to moderately large values of the capillary Reynolds number, $C^{-1} \leqslant 50$, because the computations are seemingly too expensive for larger values of $C^{-1}$. precisely when the results in Ref. 19 apply. Nonlinear vibrations produce secondary streaming flows with interesting properties that may be used to counterbalance thermocapillary convection in nonisothermal conditions. These flows have been recently analyzed both experimentally ${ }^{20,21}$ and theoretically. ${ }^{10,22,23}$
Chaotic dynamics is to be expected in periodically forced liquid bridges for appropriate values of the vibrating parameters: in fact, if forcing is sufficiently high, then the effective Reynolds number associated with both the oscillating flow and the streaming flow are also high, and both flows should exhibit some kind of turbulence. When trying to predict chaos by analytical means at the weakly nonlinear level, we are led to consider small-amplitude, nearly resonant oscillations in nearly inviscid bridges. In the absence of internal resonances. we must consider two cases. In the axisymmetric case the oscillating flow is decoupled from the streaming flow, and its complex amplitude is given by an ODE whose solution converges to a steady state (corresponding to a periodic oscillation of the bridge) for large time. ${ }^{19}$ The streaming flow is given by the usual continuity and momentum equations. with appropriate boundary conditions depending on the complex amplitude of the oscillating flow: ${ }^{19}$ even though the latter exhibits simple dynamics, the effective Reynolds numbers associated with the former may be quite large, and chaotic dynamics is to be expected, but its description relies on numerical computations. In the nonoxisymmetric case, instead, the oscillating flow is coupled with the streaming flow at leading order, and both flows must be analyzed simultaneously. They are given by a pair of ODEs (giving the complex amplitudes of a pair of counter-rotating waves associated with the oscillating flow) and a set of threedimensional (3-D) continuity and momentum equations (with appropriate boundary conditions) giving the streaming velocity and the associated averaged pressure. ${ }^{24}$ That set of coupled equations is rich enough to expect complex dynamics of the oscillating flow. but its analysis requires massive 
numerical computations (though some limited predictions can be made analytically ${ }^{24}$ ). Thus, in the absence of internal resonances. complex dynamics of the oscillating flow either is absent or requires a quite involved analysis.

The situation is completely different when internal resonances come into play because they are (by today well) known to produce chaotic dynamics in nearly conservative systems. The simplest one is the second-order $2: 1$ resonance that appears when two natural inviscid frequencies are such that $\Omega_{2}=2 \Omega_{1}$. That resonance has been recently analyzed ${ }^{25}$ for a related problem, namely. for a periodically forced bubble. The main object of this paper is precisely to analyze that resonance in axisymmetric capillary bridges, which exhibit the resonance for several values of the slenderness $\Lambda$ in the interval $0<\Lambda<\pi$ (to avoid the Rayleigh instability); see Fig. 2. We shall see that the associated oscillating flow is decoupled from the streaming flow in first approximation. and that it is given by two coupled ODEs for the slowly varying complex amplitudes of the (two) excited modes. These equations are balances of terms that account for inertia, damping, detuning from resonance, quadratic-coupling nonlinearities, and forcing. They are somewhat nonstandard because, in order to obtain quantitatively good results for realistically large values of the capillary Reynolds number, a two-term approximation is used for the damping rate; this is in accordance with the linear analysis in Ref. 16. Also, the coefficient of the quadratic nonlinearity vanishes unless a certain symmetry requirement holds. That condition is satisfied only by two resonances if only the first eight modes are considered. and takes place at $\Lambda=0.249$ and $\Lambda \simeq 2.23$. respectively. The analysis of the remaining $2: 1$ resonances requires us to include cubic nonlinearities, whose coefficients may be calculated by a somewhat tedious procedure (alike that in Ref. 19) that will not be followed because the dynamics of the associated oscillating flow is quite simple, as will be explained in Sec. IV B. Let us point out here that the complex oscillating flow described below produces a secondary slowly varying streaming flow whose spatiotemporal dynamics must be quite rich, but the analysis of this flow is beyond the scope of this paper.

The paper is organized as follows. After formulating the problem in Sec. II, we shall derive in Sec. III the coefficients of the complex amplitude equations (or normal form) that provide the weakly nonlinear dynamics of the liquid bridge. For the sake of brevity, the derivation of the coefficients associated with damping will be omitted and their values will be taken from Ref. 16 (or from Ref. 19). The relation between the small parameters $C . \mu$ and $\delta$ [see (12)] will be established in Sec. IV in such a way that all terms in the amplitude equations are of the same order. In addition. we shall rescale the amplitude equations to minimize the number of independent parameters appearing in their coefficients. and we shall analyze the resulting equations in Sec. IV A. In particular, we shall analyze completely some bifurcation diagrams for representative values of the parameters, we shall calculate Lyapunov exponents to ascertain chaotic dynamics, and we shall identify several significantly different dynamic behaviors. Let us point out here that the amplitude equations apply only in a small neighborhood of parametric resonance and that they give only a first approximation of the exact dynamics. Nevertheless, we expect the amplitude equations to be structurally stable with respect to perturbations associated with the neglected higher-order terms. Therefore, we expect our main conclusions in Sec. IV A (concerning the sequence and type of bifurcations) to be predictions for experiments, provided that measurements are sufficiently precise and the controlling parameters (the slenderness and the forcing amplitudes and frequencies) are conveniently varied: but see remark (d) in Sec. V. Some additional 2:1 resonances giving simple dynamics will be considered in Sec. IV B and some concluding remarks will be drawn in Sec. V.

\section{FORMULATION}

In order to formulate the problem, we consider a liquid bridge of length $L$, held by surface tension between two parallel, circular, coaxial disks of equal radii $R$. The volume of the liquid equals that of the space in the cylinder bounded by the disks. In addition, we assume that the density $\rho$ and the kinematic viscosity $\nu$ of the liquid, and the surface tension of the interface $\sigma$ are constant, and such that the capillary Revnolds number, $C^{-1}=\left(\sigma R / \rho \nu^{2}\right)^{1 / 2}$, is large $(C$ is also called a capillary number or an Ohnesorge number). The gravitational Bond number, $B=\rho g R^{2} / \sigma \quad(g$ = gravitational acceleration) is assumed to be small: then gravity may be neglected in the first approximation. Finally. the free surface of the liquid is anchored at the borders of the disks, and the disks are vibrating independently in the axial direction. We use the radius $R$ and the capillary time $\left(\rho R^{3} / \sigma\right)^{1 / 2}$ as the characteristic length and time for nondimensionalization to write the governing equations (continuity and momentum conservation) and boundary conditions (nonslipping at the disks, smoothness of the pressure. and velocity fields at the axis of symmetry, kinematic compatibility, and tangential and normal stress balances at the free surface) as

$$
\begin{aligned}
& u_{r}+r^{-1} u+w_{z}=0 \text {, } \\
& u_{t}+w\left(u_{z}-w_{r}\right)=-q_{r}+C\left(u_{r r}+r^{-1} u_{r}-r^{-2} u+u_{z z}\right), \\
& w_{t}+u\left(w_{r}-u_{z}\right)=-q_{z}+C\left(w_{r r}+r^{-1} w_{r}+w_{z z}\right) \text {, } \\
& u=0 . \quad w=h_{ \pm}^{\prime}(t), \quad f=\mathbf{I}, \quad \text { at } z= \pm \Lambda+h_{ \pm}(t), \\
& u=w_{r}=q_{r}=0, \quad \text { at } r=0 . \\
& u=f_{t}+f_{z} w, \quad \text { at } r=f \text {, } \\
& \left(w_{r}+u_{z}\right)\left(\mathbf{I}-f_{z}^{\hat{2}}\right)+2\left(u_{r}-w_{z}\right) f_{z}=0, \quad \text { at } r=f \text {, } \\
& q-\frac{u^{2}+w^{2}}{2}+\frac{f f_{z z}-1-f_{z}^{2}}{f\left(\mathbf{1}+f_{z}^{2}\right)^{3 / 2}} \\
& =2 C \frac{u_{r}-\left(w_{r}+u_{z}\right) f_{z}+w_{z} f_{z}^{2}}{1+f_{z}^{2}}, \quad \text { at } r=f \text {, }
\end{aligned}
$$

with appropriate initial conditions. We are using a cylindrical coordinate system $(r, \theta, z)$, with an origin midway between the disks, the axis of symmetry as the $z$ axis, and associated unit vectors $\mathbf{e}_{r}, \mathbf{e}_{\theta}$, and $\mathbf{e}_{z}$. The velocity field is $u \mathbf{e}_{r}+w \mathbf{e}_{z}$, and, for convenience, the problem is written in terms 
of the stagnation pressure, $q=$ pressure $+\left(u^{2}+w^{2}\right) / 2$ : the shape of the interface is $r=f(z, t)$ and $\Lambda=L / 2 R$ is the stenderness of the bridge. which will be assumed to be smaller than $\pi$ to avoid the Rayleigh instability. Notice that the total volume is conserved [as a consequence of (1). (4)-(6)] and. according to the assumption above, equals $2 \pi \Lambda$. i.e.,

$$
\int_{-\Lambda+h_{-}}^{\Lambda+h_{+}} f(z, t)^{2} d z=2 \Lambda
$$

Finally, the forcing functions $h_{ \pm}$are assumed to be as given by

$$
\begin{aligned}
h_{ \pm}(t)= & \mu\left[\beta_{1}^{ \pm} \exp \left(i\left(\Omega_{1}+\delta \omega_{1}\right) t\right)\right. \\
& \left.+\beta_{2}^{ \pm} \exp \left(i\left(\Omega_{2}+\delta \omega_{2}\right) t\right)+\text { c.c. }\right],
\end{aligned}
$$

where c.c. stands hereafter for the complex conjugate, and $\Omega_{1}>0$, and

$$
\Omega_{2}=2 \Omega_{1}>0
$$

are natural frequencies of the bridge in the inviscid limit; $\beta_{1}^{ \pm}$ and $\beta_{2}^{ \pm}$are complex parameters, and $\omega_{1}, \omega_{2}, \mu>0$, and $\delta$ $>0$ are real parameters. We shall consider the limit

$$
\begin{aligned}
& \left|\beta_{1}^{ \pm}\right| \sim\left|\beta_{2}^{ \pm}\right| \sim\left|\omega_{1}\right| \sim\left|\omega_{2}\right| \sim 1 . \\
& C \ll 1 . \quad \mu \ll \mathbf{I}, \quad \text { and } \delta \ll 1,
\end{aligned}
$$

without making at this stage further assumptions concerning the relative orders of magnitude of the small parameters $C$, $\mu_{\text {. }}$ and $\delta$. which will be selected in Sec. IV.

\section{AMPLITUDE EQUATIONS AND SOLVABILITY CONDITIONS}

Let us consider a particular value of the slenderness. $\Lambda$ $=\Lambda_{0}$. such that two natural frequencies in the inviscid limit satisfy (11). As we shall see at the end of this section. there are several such values of $\Lambda_{0}$ in the interval $0 \leqslant \Lambda_{0}<\pi$ involving the first eight inviscid eigenmodes. The slenderness of the capillary bridge will be considered to be appropriately close to $\Lambda_{0}$. i.e... such that

$$
\Lambda=\Lambda_{0}+\delta l, \quad \text { with }|l| \sim 1,
$$

and. in addition to the fast time variable $t \sim 1$, we shall consider the slow time variable $\tau \sim 1$, defined as

$$
\tau=\delta t
$$

where the small parameter $\delta$ is as defined in (10).

In the limit (12) we shall consider solutions of (1)-(8) that are close to the static steady state $u \equiv w \equiv q-1 \equiv 0, f$ $-1 \equiv 0$. Since the capillary Reynolds number $C^{-1}$ is large, we are led to consider four distinguished regions in the capillary bridge (see Fig. 1): (a) Two Stokes boundary lavers near the disks: (b) an interface boundary loyer near the free surface; (c) two corner tori near the edge of the disks: and (d) the bulk, that is the remaining part of the capillary bridge. The characteristic size of regions (a), (b), and (c) (where inertia and viscous terms are comparable in momentum conservation equations) is readily seen to be of the order of $\sqrt{C}$; viscous terms are negligible as compared to inertia in the bulk.

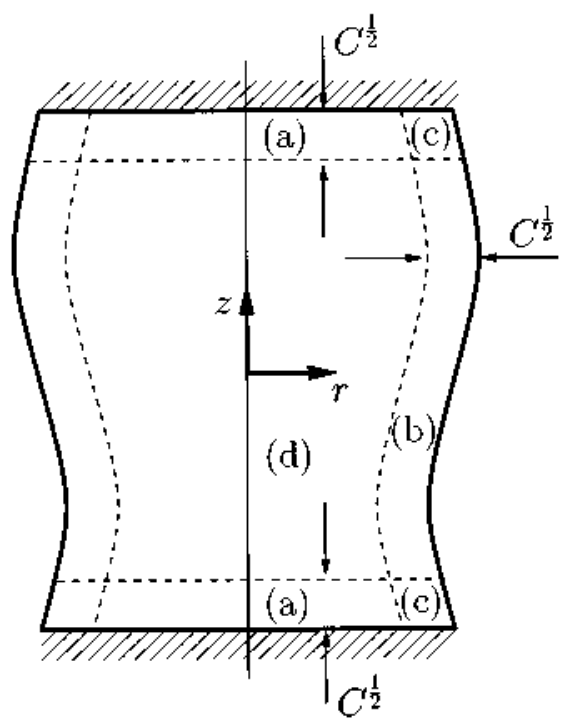

FIG. 1. The four asymptotic regions in the liquid bridge.

The solution in the bulk may be written as

$$
\begin{aligned}
u= & \epsilon \sum_{k=1}^{2} A_{k} U_{k} e^{i \Omega_{k} t}+\text { c.c. }+\mu u_{1}+\epsilon^{2} u_{2}+\delta \epsilon u_{3} \\
& +\epsilon \sqrt{C} u_{4}+\epsilon C u_{5}+\text { HOT, } \\
w= & \cdots, \quad q-1=\cdots, \quad f-1=\cdots,
\end{aligned}
$$

where the expansions for $w, q-1$ and $f-1$ are completely similar to that for $u$, and hereafter HOT stands for higherorder terms (than those displayed). For $k=1$ and 2 $\left(U_{k}, W_{k}, Q_{k}, F_{k}\right)$ is a nontrivial eigenmode associated with the eigenfrequency $\Omega_{k}$ and $A_{k}$ is the corresponding complex amplitude, which is a slowly varying function of time (i.e., depends only on the slow time variable $\tau$ ). The small parameters $\delta$ and $\mu$ are as in (10), (13), and (14), and $\epsilon$ is a real parameter, such that

$$
0<\epsilon \ll 1 \text {. }
$$

The small parameter $\epsilon$, giving the order of magnitude of the (leading-order terms in the) solution (15), will be related to $C, \delta$, and $\mu$ when analyzing the amplitude equations, in Sec. IV. In order to define the complex amplitudes we must impose an additional condition at each asymptotic order. If we impose

$$
\begin{aligned}
& \int_{0}^{2 \pi / \Omega_{k}} \int_{-\Lambda_{0}}^{\Lambda_{0}} \int_{0}^{1}\left(U_{k} u_{j}+W_{k} w_{j}\right) e^{-i \Omega_{k^{t}} r d r d z d t} \\
& =0 . \quad \text { for } k=1.2, \quad \text { and all } j \geqslant 1 .
\end{aligned}
$$

then $A_{k}$ is defined as $\epsilon A_{k}=\Omega_{k} \iiint\left(U_{k} u\right.$ $\left.+W_{k} w\right) e^{-i \Omega_{k} t} r d r d z d t /\left[2 \pi \iint\left(U_{k}^{2}+W_{k}^{2}\right) r d r d z\right]$, for $k=1$ and 2 .

The complex amplitudes, $A_{1}$ and $A_{2}$, are seen to satisfy the following amplitude equations: 


$$
\begin{aligned}
\epsilon \delta d A_{k} / d \tau= & -\left[(1+i) \alpha_{4 k} \sqrt{C}+\alpha_{5 k} C-i \alpha_{3 k} / \delta\right] \epsilon A_{k} \\
& +i \alpha_{2 k} \epsilon^{2} N_{k}+i \mu\left(\alpha_{1 k}^{+} \beta_{k}^{+}-\alpha_{1 k}^{-} \beta_{k}^{-}\right) e^{i \omega_{k} \tau} \\
& + \text { HOT }
\end{aligned}
$$

for $k=1$ and 2, where the quadratic nonlinearities $N_{1}$ and $N_{2}$ are

$$
N_{1}=A_{2} \overline{A_{1}} . \quad N_{2}=A_{1}^{2} .
$$

with the overbar standing for the complex conjugate. The parameters $\epsilon, \delta, l, \mu, \beta_{k}^{ \pm}$and $\omega_{k}$ are as defined in $(10),(13)$. and (15)

The terms displayed in Eqs. (18) account for inertia, damping, detuning from resonance, nonlinearity, and forcing and (up to rescaling) essentially amount to the usual normal form that applies to this internal resonance. Their specific feature here is that they include a two-term approximation to the damping rate, $\alpha_{4 k} \sqrt{C}+\alpha_{5 k} C$. In so doing, we are anticipating that $\alpha_{4 k}$ is typically about $10^{-2}$ times $\alpha_{5 k}$ : thus. if only the leading-order term were considered, then the resulting approximation would be useful only for extremely small values of $C$ (roughly $C \leqslant 10^{-5}$ ) while the two-term approximation gives reasonably good results for $C \leq 10^{-2}$. A straightforward orders-of-magnitude analysis readily shows that the first term, $\alpha_{4 k} \sqrt{C}$, accounts for viscous dissipation in the Stokes boundary layers and the second term, $\alpha_{5 k} C$, comes from viscous dissipation in the bulk and a first correction of viscous dissipation in the Stokes boundary layers: viscous dissipation in the interface boundary layer and in the corner tori provides higher-order terms [of orders $\epsilon \mathrm{C}^{3 / 2}$ and $\epsilon(C \log C)^{2}$, respectively] in (18). Notice also that if HOT are neglected, then Eqs. (18) are decoupled from the secondary streaming flow. This is so because the streaming velocity is roughly of the order of $\epsilon^{2}$ (see Ref. 19) and thus its effect on (18) cannot be larger than $\epsilon^{3}$ (in fact, it is even smaller than that because, the oscillating flow being axisymmetric, the leading $\epsilon^{3}$ effect identically vanishes, as in the analysis in Ref. 19).

The coefficients $\alpha_{1 k}^{ \pm}$and $\alpha_{j k}$ (for $j=2, \ldots, 5$ and $k=1$ and 2) appearing in (18) are real and may be calculated as follows. Substitute (10), (13)-(15), and (18) into (1)-(3) and (5) and set to zero the coefficients of $\epsilon A_{1}, \epsilon A_{2}, \mu, \epsilon^{2}, \epsilon \delta$, $\epsilon \sqrt{C}$. and $\epsilon C$, to obtain a system of seven equations and boundary conditions at $r=0$ for $\left(U_{k}, W_{k}, Q_{k}, F_{k}\right)$ and $\left(u_{j}, w_{j}, q_{j}, f_{j}\right)$ for $k=1$ and 2 and $j=1 \ldots, 5$ : the corresponding boundary conditions at the edge of the Stokes and the interface boundary layers (i.e., at $z= \pm \Lambda_{0}$ and at $r=1$, in first approximation) are obtained from a (somewhat tedious) analysis of the layers, through the appropriate matching conditions. Then the coefficients $\alpha_{1 k}^{ \pm}$and $\alpha_{j k}$ are obtained by applying the appropriate solvability conditions [to the problems giving $\left(u_{j}, w_{j}, q_{j}, f_{j}\right)$ for $\left.j=1, \ldots, 5\right]$. But, if proceeding in this standard way, some care must be taken with a singularity near the edge of the disks. $r=1, z= \pm \Lambda_{0}$, which may lead to wrong results at order $\epsilon C$ if not appropriately handled ${ }^{16}$ (see also Refs. 26 and 27 for earlier analyses of this difficulty in related problems). An alternative way to calculating the coefficients of the amplitude equation was given in Ref. 19, where an integral solvability condition applied to the original problem (1)-(8) was presented that does not require special care with the above-mentioned singularity. But, for the sake of brevity, we shall not give here the detailed calculation of the coefficients $\alpha_{4 k}$ and $\alpha_{5 k}$, which are associated with the linear response of the bridge and thus they are not specific of the parametric resonance we are analyzing. Instead. these coefficients may be calculated once forever, as it was done for a related normal form in Ref. 19 (or in a strictly linear analysis. in Ref. 16). The expressions for these two coefficients are given in Appendix $A$ [in Eqs. (A5)-(A6)]

Now the [inviscid eigenfrequencies $\Omega_{k}$ and eigenmodes ( $U_{k}, W_{k}, Q_{k}, F_{k}$ ). and the] remaining coefficients in Eqs. (18), $\alpha_{1 k}^{ \pm}, \alpha_{2 k}$, and $\alpha_{3 k}$, are calculated below by first setting $C=0$ in (1)-(8) (then the thin viscous oscillatory boundary layers disappear), replacing (10), (13)-(15), and (18) into the resulting equations and boundary conditions [and in condition (9) that must be also used] and setting to zero the coefficients of $\epsilon A_{1}, \epsilon A_{2}, \mu, \epsilon^{2}$, and $\epsilon \delta$. Then we obtain the problems (20)-(25) and (26) $-(31)$ below and a solvability condition applied to the latter, for $j=1,2$, and 3 , provides the coefficients. But some care must be taken when doing that because the limit $C \rightarrow 0$ involves a singular perturbation and does not necessarily commute with the limit $\epsilon \rightarrow 0, \mu \rightarrow 0, \delta \rightarrow 0$. In fact, both limits do not commute, in general; streaming flows driven by oscillatory boundary layers (whose driving mechanism disappears if $C=0$ but is independent of $C$ in first approximation if $C$ is small but nonzero ${ }^{28}$ ) are good examples; see Refs. 19 and $22-24$ for the analysis of such flows in capillary bridges. Fortunately. that difficulty is absent in our case, as it comes out from the analysis (that is omitted for the sake of brevity and is similar to that in Ref. 19) of the Stokes and the interface boundary layers, which are seen to add nothing to the boundary conditions at the disks and the interface, at orders $\epsilon, \mu, \epsilon^{2}$, and $\epsilon \delta$.

If we proceed as indicated in the last paragraph, then we obtain the following problems at orders $\epsilon . \mu . \epsilon^{2}$, and $\epsilon \delta$.

$$
\begin{aligned}
& U_{k r}+r^{-1} U_{k}+W_{k z}=0, \\
& i \Omega_{k} U_{k}+Q_{k r}=i \Omega_{k} W_{k}+Q_{k z}=0, \\
& W_{k}=0, \quad F_{k}=0, \quad \text { at } z= \pm \Lambda_{0}, \\
& U_{k}=W_{k r}=0, \quad \text { at } r=0, \\
& U_{k}-i \Omega_{k} F_{k}=Q_{k}+F_{k}+F_{k}^{\prime \prime}=0, \quad \text { at } r=1, \\
& \int_{-\Lambda_{0}}^{\Lambda_{0}} F_{h}(z) d z=0,
\end{aligned}
$$

for $k=1$ and 2 , and

$$
\begin{aligned}
& u_{j r}+r^{-1} u_{j}+w_{j z}=0, \\
& u_{j t}+q_{j r}+g_{j}^{1}=w_{j t}+q_{j z}+g_{j}^{2}=0 . \\
& w_{j}=G_{j}^{ \pm}, \quad f_{j}=L_{j}^{ \pm}, \quad \text { at } z= \pm \Lambda_{j}, \\
& u_{J}=w_{j r}=0, \quad \text { at } r=0,
\end{aligned}
$$




$$
\begin{aligned}
& u_{j}-f_{j t}-H_{j}^{1}=q_{j}+f_{j}+f_{j z z}-H_{j}^{2}=0, \quad \text { at } r=\mathbf{l} . \\
& \int_{-\Lambda_{0}}^{\Lambda_{0}} f_{j} d z+M_{j}=0,
\end{aligned}
$$

for $j=1,2$, and 3 , where the forcing terms $g_{j}^{p}, G_{j}^{ \pm}, L_{j}^{ \pm}$, $H_{j}^{p}$, and $M_{j}$, for $p=1$ and 2, are given in Appendix A [in Eqs. (A8)-(A22), at the end of the paper].

The linear eigenvalue problem $(20)-(25)$ provides the natural frequencies $\Omega_{k}$ and the associated eigenmodes. A fairly simple semianalytical solution to that problem is given in Appendix A [in Eqs. (A1)-(A4), at the end of the paper], as taken from Ref. 13. From this solution we see that there are two kinds of inviscid modes. Odd modes are obtained when $\Omega_{k}$ is a solution of the first equation (A1), and then $U_{k}, z W_{k}, Q_{k}$, and $F_{k}$ are odd functions of the $z$ variable. If. instead. $\Omega_{k}$ satisfies the second equation (A1), then we obtain the even modes and $U_{k}, z W_{k}, Q_{k}$, and $F_{k}$ are even functions of $z$. Equations (A2)-(A4) also show that the eigenfunctions (i.e.. the common constant factor) may be always selected, such that $Q_{k}$ and $F_{k}$ are real, and $U_{k}$ and $W_{k}$ are purely imaginary.

Now the coefficients $\alpha_{1 k}^{ \pm}, \alpha_{2 k}$, and $\alpha_{3 k}$ of the amplitude equations will be calculated below from the solvability condition for $(26)-(31)$ that is obtained by requiring the timedependent solution to that problem to be bounded in the fast time scale (i.e.. as $t \rightarrow \infty$ ), as follows. Multiply the first and second equations $(21)$ by $-r u_{j} e^{-i \Omega_{k^{t}}}$ and $-r w_{j} e^{-i \Omega_{k^{t}}}$. respectively, and the first and second equations (27) by $r U_{k} e^{-i \Omega_{k} t}$ and $r W_{k} e^{-i \Omega_{k^{t}}}$, respectively, add, integrate in 0 $<r<1,-\Lambda_{0}<z<\Lambda_{0}$, integrate by parts, and take into account the continuity equations $(20)$ and $(26)$, and the boundary conditions $(22)-(24)$ and $(28)-(30)$, to obtain

$$
d I_{k j}^{1} / d t=I_{k j}^{\hat{2}} e^{-j \Omega_{k^{t} t}}
$$

for $k=1$ and 2 and $j=1,2$ and 3 , where

$$
\begin{aligned}
I_{k j}^{1}= & e^{-i \Omega_{k^{t}}}\left[\int_{-\Lambda_{0}}^{\Lambda_{0}} \int_{0}^{1}\left(U_{k} u_{j}+W_{h} w_{j}\right) r d r d z\right. \\
& \left.+\int_{-\Lambda_{0}}^{\Lambda_{0}}\left(F_{k}+F_{k}^{\prime \prime}\right) f_{j} d z\right] . \\
I_{k j}^{2}= & -\int_{-\Lambda_{0}}^{\Lambda_{0}} \int_{0}^{1}\left(U_{k} g_{J}^{1}+W_{k} g_{j}^{2}\right) r d r d z \\
& +\int_{0}^{1}\left[Q_{k}\left(r, \Lambda_{0}\right) G_{J}^{+}(r)-Q_{k}\left(r_{*}-\Lambda_{0}\right) G_{J}^{-}(r)\right] r d r \\
& -\int_{-\Lambda_{0}}^{\Lambda_{0}}\left[\left(F_{k}+F_{k}^{\prime \prime}\right) H_{J}^{1}+i \Omega_{k} F_{k} H_{j}^{2}\right] d z \\
& -i \Omega_{k}\left[F_{k}^{\prime}\left(\Lambda_{0}\right) L_{j}^{+}-F_{k}^{\prime}\left(-\Lambda_{0}\right) L_{j}^{-}\right] .
\end{aligned}
$$

Equation (32) shows that (i) if $u_{j}, w_{j}$, and $f_{j}$ are bounded as $t \rightarrow \infty$ then $\left|\int_{0}^{t} I_{k j}^{2} e^{-i \Omega_{k} t} d t\right|$ is also bounded as $t \rightarrow \infty$. and (ii) this integral is convergent if and only if the resonant part of $I_{k j}^{2}$ [i.e., that part of $I_{k j}^{2}$ that depends on the fast time variable as $\left.\exp \left(i \Omega_{k} t\right)\right]$ is identically zero for $k=1$ and 2 and $j=1,2$, and 3. These (six) conditions provide the coefficients $\alpha_{1 k}^{ \pm}$, $\alpha_{2 k}$, and $\alpha_{3 k}$, which are found to be

$$
\begin{aligned}
& 2 \alpha_{1 k}^{ \pm} \int_{-\Lambda_{0}}^{\Lambda_{0}}\left(F_{k}^{2}-F_{k}^{\prime 2}\right) d z=\Omega_{k} \int_{0}^{1} Q_{k}\left(r_{*} \pm \Lambda_{0}\right) r d r \\
& 2 \alpha_{21} \int_{-\Lambda_{0}}^{\Lambda_{0}}\left(F_{1}^{2}-F_{1}^{\prime^{2}}\right) d z / \Omega_{1} \\
& =2 \alpha_{22} \int_{-\Lambda_{0}}^{\Lambda_{0}}\left(F_{2}^{2}-F_{2}^{\prime 2}\right) d z / \Omega_{1} \\
& =\int_{-\Lambda_{0}}^{\Lambda_{0}}\left[3\left(\Omega_{1}^{2}-1\right) F_{1}^{2} F_{2}+F_{1}^{\prime 2} F_{2}+2 F_{1} F_{1}^{\prime} F_{2}^{\prime}\right. \\
& \left.+\left(2 F_{1} W_{1} W_{2}-F_{2} W_{1}^{2}\right)_{r=1}\right] d z . \\
& \alpha_{3 k} \int_{-\Lambda_{0}}^{\Lambda_{0}}\left(F_{k}^{2}-F_{k}^{\prime 2}\right) d z \\
& =-\int_{0}^{1} r Q_{k r}\left(r, \Lambda_{0}\right)^{2} d r / \Omega_{k}+\Omega_{k} F_{k}^{\prime}\left(\Lambda_{0}\right)^{2},
\end{aligned}
$$

for $k=1$ and 2 . In order to obtain these expressions we have integrated by parts repeatedly, and used (11). (20)-(25). (A8)-(A22) (in Appendix A), and the following equations:

$$
\begin{aligned}
\int_{-\Lambda_{0}}^{\Lambda_{0}} \int_{0}^{1}\left(U_{k}^{2}+W_{k}^{2}\right) r d r d z & =\int_{-\Lambda_{0}}^{\Lambda_{0}}\left(F_{k}+F_{k}^{\prime \prime}\right) F_{k} d z \\
& =\int_{-\Lambda_{0}}^{\Lambda_{0}}\left(F_{k}^{2}-F_{k}^{\prime 2}\right) d z
\end{aligned}
$$

which are readily obtained upon multiplication of the first and second equations (2I) by $r U_{k}$ and $r W_{k}$, respectively. integration in $0<r<1$ and $-\Lambda_{0}<z<\Lambda_{0}$. integration by parts and substitution of $(22)-(24)$.

The amplitude equations (18). with the coefficients as calculated from (33)-(35) and (A5)-(A6) (in Appendix A) will be thoroughly analyzed in Sec. IV. At the moment, several comments concerning the coefficients are in order.

(a) If, according to our comment above, the eigenfunctions associated with $\Omega_{1}$ and $\Omega_{2}$ are selected such that $i U_{k}$, $i W_{k}, Q_{k}$, and $F_{k}$ are real for $k=1$ and 2 , then $\alpha_{1 k}^{ \pm}, \ldots, \alpha_{5 k}$ are real [see (33)-(35) and (A5)-(A6)] as anticipated above.

(b) The coefficients associated with the forcing term, $\alpha_{1 k}^{ \pm}$, satisfy

$$
\alpha_{1 k}^{-}=-\alpha_{1 k}^{+} \text {or } \quad \alpha_{1 k}^{-}=\alpha_{1 k}^{+},
$$

depending on whether the eigenmode associated with $\Omega_{k}$ is odd or even, respectively, as readily seen from (33).

(c) If the eigenmode associated with $\Omega_{2}$ is odd, then $\alpha_{21}=\alpha_{22}=0$, as readily seen from (34). In this case, which will be briefly considered in Sec. III B, cubic terms must be added to the amplitude equations.

(d) If, instead, the eigenmode associated with $\Omega_{2}$ is even, then none of the coefficients associated with quadratic terms vanishes generically, and the amplitude equations (18) apply to give the dynamics of the oscillating flow. Still, in this case, $\alpha_{21}$ and $\alpha_{22}$ have the same sign: this is readily seen in (34) when taking into account that $\Omega_{2}=2 \Omega_{1}>0$, and that $\int_{-\Lambda_{0}}^{\Lambda_{0}}\left(F_{k}^{2}-F_{k}^{\prime 2}\right) d z<0$ for $k=1$ and 2 , as it comes out from 


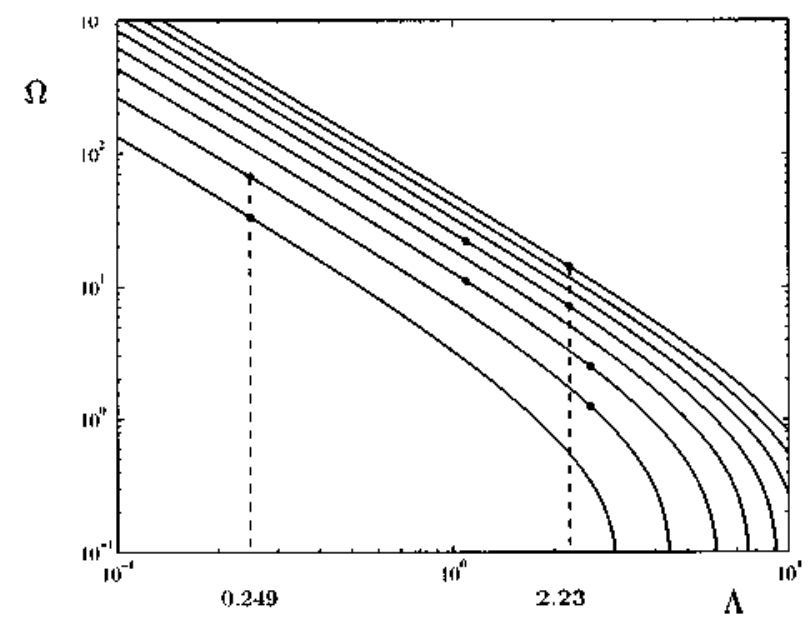

FIG. 2. The eigenfrequencies of the first eight inviscid eigenunodes in terms of the slenderness. The internal 2:1 resonances in the interval $0<\Lambda<\pi$ are indicated with dots.

the variational definition of the first eigenvalue of $\varphi^{\prime \prime}(z)$ $+\varphi(z)+\lambda \varphi(z)=0$ in $-\Lambda_{0}<z<\Lambda_{0}, \varphi\left( \pm \Lambda_{0}\right)=0$, which is strictly positive if $\Lambda_{0}<\pi$.

(e) The eigenfrequencies of the first eight inviscid eigenmodes are plotted in Fig. 2 in terms of $\Lambda$. as calculated from the two equations in (A1) (in the Appendix). The (four) 2:1 resonances involving these modes in the interval $0<\Lambda_{0}$ $<\pi$ are indicated with dots. Notice that only two of them (namely. those at $\Lambda_{0} \simeq 0.249$ and $\Lambda_{0} \simeq 2.23$ ) are such that the higher eigenfrequency corresponds to an even mode [and thus the quadratic coefficients are nonzero, according to remarks (c) and (d) above]. For those two resonances we have

$$
\begin{aligned}
& \Lambda_{0} \simeq 249, \quad \Omega_{2}=2 \Omega_{1} \simeq 66.76, \quad \alpha_{11}^{+}=-\alpha_{11}^{-} \simeq 159.5, \\
& \alpha_{12}^{+}=\alpha_{12}^{-} \simeq-652.0 . \quad \alpha_{21} \simeq 11.239, \quad \alpha_{22} \simeq 10.712 . \\
& \alpha_{31} \simeq-207.89, \quad \alpha_{32} \simeq-408.16, \quad \alpha_{41} \simeq 3.197, \\
& \alpha_{42} \simeq 3.269, \quad \alpha_{51} \simeq 145.8, \quad \alpha_{52} \simeq 443.9,
\end{aligned}
$$

and

$$
\begin{aligned}
& \Lambda_{0} \simeq 2.23, \quad \Omega_{2}=2 \Omega_{1} \simeq 14.14, \quad \alpha_{11}^{+}=-\alpha_{11}^{-} \simeq 3.362 . \\
& \alpha_{12}^{+}=\alpha_{12}^{-} \simeq-8.054, \quad \alpha_{21} \simeq 0.699, \quad \alpha_{22} \simeq 0.578, \\
& \alpha_{31} \simeq-5.294, \quad \alpha_{32} \simeq-10.02, \quad \alpha_{41} \simeq 0.083, \\
& \alpha_{42} \simeq 0.158, \quad \alpha_{51} \simeq 25.46 . \quad \alpha_{52} \simeq 64.58 .
\end{aligned}
$$

\section{DYNAMICS OF THE CAPILLARY BRIDGE}

As seen in Sec. III, the dynamics of the oscillating flow associated with this internal resonance is given by Eq. (18), with the real coefficients $\alpha_{1 k}^{ \pm}, \alpha_{2 k}, \ldots, \alpha_{5 k}$ as given by (33)(35) and (A5)-(A6). According to remarks (c) and (d) at the end of Sec. III, we are led to consider two cases

\section{A. Quadratic nonlinearity $\left(\Omega_{2}\right.$ associated with an even mode)}

If $\Omega_{2}$ is associated with an even mode (as in the two cases considered at the end of Sec. III) then the coefficients of the nonlinear, quadratic terms in (18) are nonzero. Then quadratic nonlinearities saturate the weakly nonlinear dynamics, and we can relate the small parameters $\epsilon, \delta, \mu$, and $C$ such that all terms explicitly displayed in (18) are comparable. To this end and to minimize the number of independent parameters in the final equations, we redefine the small parameters $\epsilon, \delta$, and $\mu$, and the complex amplitudes $A_{1}$ and $A_{2}$ as follows:

$$
\begin{aligned}
& \epsilon=\delta=\alpha_{42} \sqrt{C}+\alpha_{52} C . \quad \mu=\epsilon^{2} /\left(\alpha_{11}^{+} \sqrt{\alpha_{21} \alpha_{22}}\right), \\
& A_{1}=\left(\alpha_{21} \alpha_{22}\right)^{-1 / 2} B_{1} e^{j \omega \tau} . \quad A_{2}=\alpha_{21}^{-1} B_{2} e^{2 i \omega \tau} .
\end{aligned}
$$

to rewrite the amplitude equations (18) as

$$
d B_{1} / d \tau=-(a+i \tilde{\omega}) B_{1}+i \overline{B_{1}} B_{2}+i\left(\beta_{1}^{+}+\beta_{1}^{-}\right) e^{i\left(\omega_{1}-\omega\right) \tau}
$$

$$
\begin{aligned}
d B_{2} / d \tau= & -[1+2 i(\widetilde{\omega}+\widetilde{l})] B_{2}+i B_{1}^{2} \\
& +i b\left(\beta_{2}^{+}-\beta_{2}^{-}\right) e^{i\left(\omega_{2}-2 \omega\right) \tau} .
\end{aligned}
$$

where we have neglected higher-order terms. and. since we are going to consider only the two cases at the end of Sec. II. we are taking into account that $\alpha_{11}^{+}=-\alpha_{11}^{-}$and $\alpha_{12}^{+}=\alpha_{12}^{-}$. Notice that $\delta$ equals the damping rate (due to viscous dissipation) of the eigenmode associated with $\Omega_{2}$, and thus the slow time scale for the evolution of the complex amplitudes is a viscous time scale. The constant $\omega$ will be selected below such that either $\omega=\omega_{1}$ or $\omega=\omega_{2} / 2$. and the parameters $a, b$. $\widetilde{\omega}$, and $\widetilde{l}$, appearing in $(40)-(4 I)$ are

$$
\begin{aligned}
& a=\left(\alpha_{41}+\alpha_{51} \sqrt{C}\right) /\left(\alpha_{42}+\alpha_{52} \sqrt{C}\right), \\
& b=\left(\alpha_{12}^{+} / \alpha_{11}^{+}\right) \sqrt{\alpha_{21} / \alpha_{22}}, \\
& \widetilde{\omega}=\omega+\alpha_{41} \sqrt{C} / \epsilon-\alpha_{31} l, \\
& \tilde{l}=\left(\alpha_{42}-2 \alpha_{41}\right) \sqrt{C} /(2 \epsilon)+\left(2 \alpha_{31}-\alpha_{32}\right) / 2 .
\end{aligned}
$$

Notice that $b$ is a constant and $a$ depends only on $C$. which in turn is constant once the radius of the disks and the liquid are fixed. Still, in the two cases considered at the end of Sec. III, we have

$$
a \simeq 0.3284 \frac{0.0219+\sqrt{C}}{0.007364+\sqrt{C}} . \quad \text { if } \Lambda_{0} \simeq .249
$$

and

$$
a \simeq 0.3942 \frac{0.00326+\sqrt{C}}{0.00245+\sqrt{C}}, \quad \text { if } \quad \Lambda_{0} \simeq 2.23 \text {. }
$$

and thus $a$ is quite insensitive to variations of $C$ in the realistic range $10^{-4} \leq C \leq 10^{-2}$. Instead. $\tilde{\sigma}$ and $\tilde{T}$ are scaled measures of detuming from resonance that may be varied independently by choosing the forcing frequencies and the slendemess, respectively; thus they must be treated as free parameters.

Although we have derived the amplitude equations for the most general case when both resonant modes are directly excited, for the sake of brevity, we shall only analyze the 
case when only one forcing frequency is present, and all numerical applications below will be concemed with the resonance at $\Lambda_{0} \simeq 2.23$.

\section{Forcing with the higher-frequency $\Omega_{2}\left(\beta_{1}^{+}=\beta_{1}^{-}=0\right)$}

In this case we define the constant $\omega$ appearing in (39)(41) and (43) as

$\omega=\omega_{2} / 2$,

to rewrite Eqs. (40)-(41) as

$$
\begin{aligned}
& d B_{1} / d \tau=-(a+i \widetilde{\omega}) B_{1}+i \overline{B_{1}} B_{2}, \\
& d B_{2} / d \tau=-[1+2 i(\widetilde{\omega}+\widetilde{l})] B_{2}+i B_{1}^{2}+\gamma,
\end{aligned}
$$

where the scaled forcing amplitude $\gamma$ is given by

$$
\gamma=i b\left(\beta_{2}^{+}-\beta_{2}^{-}\right),
$$

and will be selected to be real and positive; this can be done because, according to $(10)$, the phase of $\beta_{2}^{+}-\beta_{2}^{-}$can be arbitrarily fixed by introducing a phase shift in the time variable. Also, if both disks are vibrated, then they must not be vibrated in phase with the same amplitude in order to avoid $\gamma$ to be identically zero. This was to be expected because the eigenmode associated with $\Omega_{2}$ is even and thus cannot be directly excited by purely antisymmetric vibrations.

The specific applications below will be done for $a$ $=0.4034$. This value of $a$ has been obtained by taking $C$ $=10^{-3}$ (a typical value of the capillary number) in the expression of $a$ [which follows from Eqs. (37) and (42)] for the resonance at $\Lambda_{0}=2.23$. Notice that anyway, $a$ is fairly independent of $C$, as indicated above

The normal form (46)-(47) is invariant under the symmetries

$$
\tilde{l} \rightarrow-\tilde{l}, \quad \widetilde{\omega} \rightarrow-\widetilde{\omega}, \quad B_{1} \rightarrow i \overline{B_{1}}, \quad B_{2} \rightarrow \overline{B_{2}},
$$

and

$$
B_{1} \rightarrow-B_{1} .
$$

The invariance under (49) implies that the dynamics of (46)(47) for $(\widetilde{l}, \widetilde{\omega})=\left(\widetilde{l}_{0}, \widetilde{\omega_{0}}\right)$ is identical to that for $(\widetilde{l}, \widetilde{\omega})$ $=\left(-\tilde{l}_{0},-\widetilde{\omega_{0}}\right)$. The symmetry $(50)$ will also play some role below: the solutions of (46)-(47) that are invariant under (50) will be called symmetric and obviously nonsymmetric solutions exist in pairs.

Equations (46)-(47) exhibit two kinds of steady states [which correspond to mono- and bichromatic oscillations of the liquid bridge; see (15) and (39)], whose amplitudes are given by

$$
\left|B_{1}^{s}\right|=0, \quad\left|B_{2}^{s}\right|^{2}=\gamma^{2} /\left[1+4\left(\widetilde{\omega}+\widetilde{I}^{2}\right],\right.
$$

and

$$
\begin{aligned}
& \left|B_{1}^{s}\right|^{2}=2 \widetilde{\omega}(\widetilde{\omega}+\widetilde{l})-a \pm \sqrt{\gamma^{2}-[\widetilde{\omega}+2 a(\widetilde{\omega}+\widetilde{l})]^{2}}, \\
& \left|B_{2}^{s}\right|^{2}=\widetilde{\omega}^{2}+a^{2},
\end{aligned}
$$

respectively. The stability criteria in Appendix B imply that the symmetric steady states (51) are stable if $\gamma<\gamma_{s 1}$ and unstable if $\gamma>\gamma_{s 1}$, where

$$
\gamma_{s 1}^{2}=\left[2 \widetilde{\omega}(\widetilde{\omega}+\widetilde{l}-a]^{2}+[\widetilde{\omega}+2 a(\widetilde{\omega}+\widetilde{l})]^{2} .\right.
$$

The branch of nonsymmetric steady states (52) bifurcates (through a pitchfork bifurcation) from the symmetric ones at $\gamma=\gamma_{s 1}$, and they depend monotonically on $\gamma$ if $\widetilde{\omega_{1}} \leqslant \widetilde{\omega}$ $\leqslant \widetilde{\omega}_{1}^{+}$, where

$$
\widetilde{\omega}_{1}^{ \pm}=\left(-\tilde{l} \pm \sqrt{l^{2}+2 a}\right) / 2 .
$$

In addition, according to the stability criteria in Appendix B, all nonsymmetric steady states are asymptotically stable if either (i) $\widetilde{l}^{2} \leqslant \widetilde{l}_{c}^{2}$ or (ii) $\widetilde{l}^{2}>\widetilde{l}_{c}^{2}$, but either $\left(\widetilde{\omega}_{1}^{-} \leqslant\right) \widetilde{\omega} \leqslant \widetilde{\omega}_{2}^{-}$or $\left(\widetilde{\omega}_{1}^{+} \geqslant\right) \widetilde{\omega} \geqslant \widetilde{\omega}_{2}^{+}$, where $\widetilde{l}_{c}, \widetilde{\omega}_{2}^{-}$, and $\widetilde{\omega}_{2}^{+}$are as defined in Eqs. (55)-(56) below. If instead, (54) holds but

$$
\widetilde{l}^{2}>\widetilde{l}_{c}^{2}=\mathbf{4} a+2 \text {. }
$$

and $\left(\widetilde{\omega}_{1}^{-}<\right) \widetilde{\omega}_{2}^{-}<\widetilde{\omega}<\widetilde{\omega}_{2}^{+}\left(<\widetilde{\omega}_{1}^{+}\right)$, where

$$
\widetilde{\omega}_{2}^{ \pm}=\left(-3 \tilde{l} \pm \sqrt{l^{2}-4 a-2}\right) / 4 \text {. }
$$

then the branch of nonsymmetric steady states exhibits a Hopf bifurcation at $\gamma=\gamma_{\mathrm{H}}$, where

$$
\gamma_{\mathrm{H}}=\varphi(\widetilde{\omega}, a, \widetilde{l}), \text { for } \widetilde{\omega}_{2}^{-}<\widetilde{\omega}<\widetilde{\omega}_{2}^{+} \text {. }
$$

and the nonsymmetric steady states are asymptotically stable for $\gamma_{s 1}<\gamma<\gamma_{\mathrm{H}}$ and unstable for $\gamma>\gamma_{\mathrm{H}}$. The function $\varphi$ is obtained upon substitution of (52) into (B2)-(B4) (in Appendix B) and solving Eq. (B6), and is such that $\gamma_{\mathrm{H}}>\gamma_{s 1}$ and $\gamma_{\mathrm{H}} \rightarrow \infty$ as $\widetilde{\omega} \rightarrow \widetilde{\omega}_{2}^{ \pm}$. The explicit expression of $\varphi$ is omitted for the sake of brevity.

If either $\widetilde{\omega}<\widetilde{\omega}_{1}^{-}$or $\widetilde{\omega}>\widetilde{\omega}_{1}^{+}$, then the branch of nonsymmetric steady states is $\mathrm{C}$ shaped, with a bending point at $\gamma$ $=\gamma_{s 2}$, where

$$
\gamma_{s 2}=|\widetilde{\omega}+2 a(\widetilde{\omega}+\tilde{l})| \quad\left(<\gamma_{s 1}\right) ;
$$

also, the stability criteria in Appendix B imply that the nonsymmetric steady states in the lower branch are unstable and those in the upper branch are stable.

Now, for a fixed value of $\tilde{\tau}$. the plot of the critical values of the scaled forcing amplitudes, $\gamma_{s 1}, \gamma_{s 2}$, and $\gamma_{\mathrm{H}}$. in terms of $\tilde{\omega}$, is always qualitatively similar to that in Fig. 3(a) if condition (55) holds; otherwise, the upper branch giving $\gamma_{\mathrm{H}}$ disappears. In the special case $\tilde{l}=0$, the plots in Fig. 3(a) are symmetric [according to the symmetry (49)] and the curve giving $\gamma_{\mathrm{H}}$ disappears [because condition (55) does not hold]. Thus, there is always a stable steady state. and (as we have checked numerically) the large-time dynamics of (46)-(47) is fairly simple.

If. both $\tilde{\sigma}$ and $\tilde{l}$ are kept fixed. then the plots of $\left|B_{1}^{s}\right|$ and $\left|B_{2}^{s}\right|$ in terms of $\gamma$ are as those in Fig. 3(b) if $\widetilde{\omega_{1}}<\tilde{\omega}$ $<\widetilde{w_{1}}$, and as those in Fig. 3(c) otherwise. Notice that as $\gamma$ increases $\left|B_{1}^{S}\right|$ dominates in the nonsymmetric branch as was to be expected [see (52)]. This remarkable well-known property implies that, for large forcing, the mode that is not directly excited dominates the other one. A similar effect is not true in the more complex time-dependent solutions considered below. If (55) holds and $\widetilde{\omega}_{2}^{-}<\widetilde{\omega}<\widetilde{\omega}_{2}^{+}$, then a Hopf bifurcation point appears, in the branch of nonsymmetric solutions, at $\gamma=\gamma_{\mathrm{H}}$, as in Figs. 3(d) and 3(e), where for the 
(a)

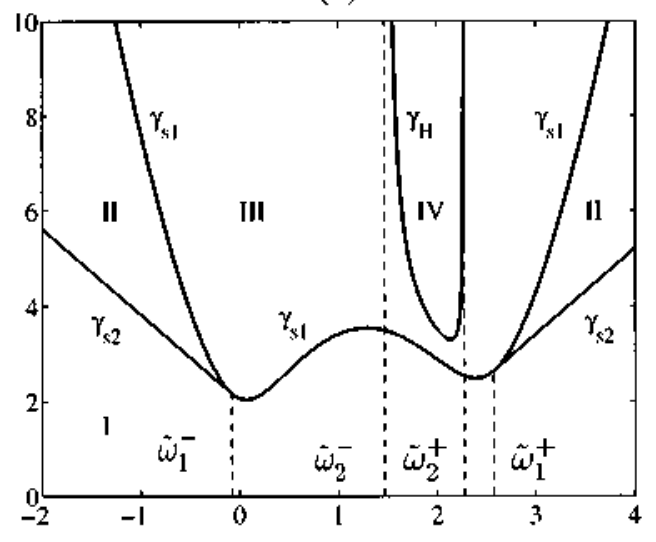

(b)

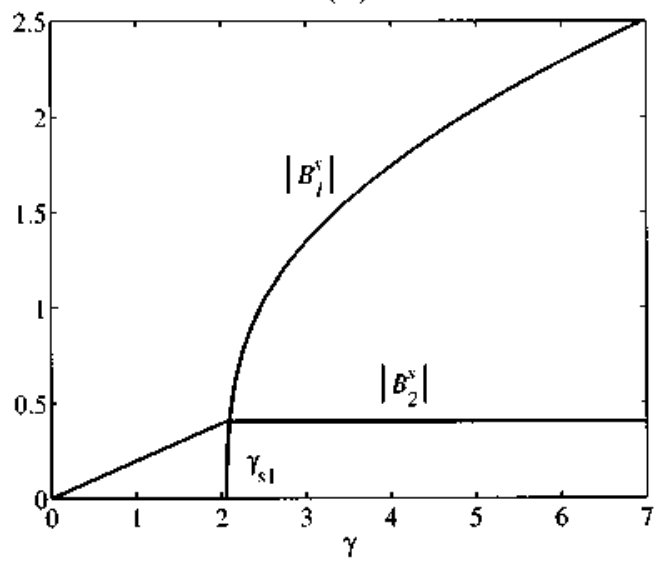

(c)

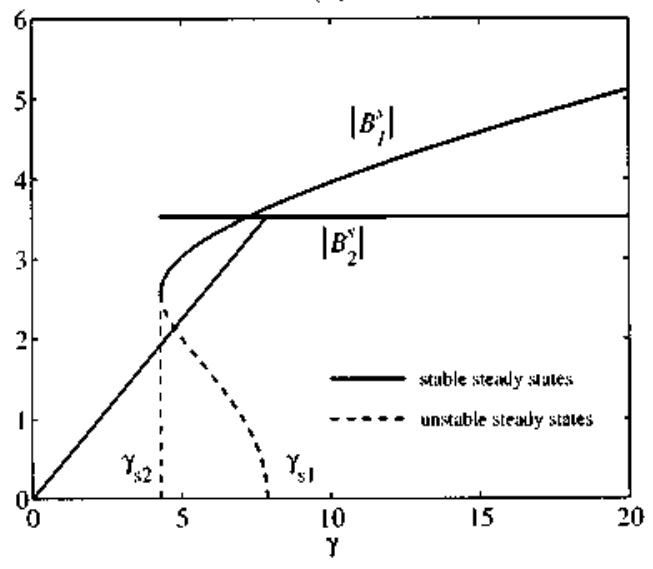

sake of clarity only $\left|B_{1}^{s}\right|$ is plotted, and for convenience a part of the bifurcated branch of periodic solutions is also plotted: notice that the Hopf bifurcation at $\gamma=\gamma_{\mathrm{H}}$ can be supercritical [as in Fig. 3(d)] or subcritical [as in Fig. 3(e)].

Now, in order to plot periodic or aperiodic attractors below, we shall consider the intersections (such that the orbit points outward) of the attractor with the Poincare hypersurface (a hyperellipsoid in the four-dimensional phase space spanned by the real and imaginary parts of $B_{1}$ and $B_{2}$ ), (d)

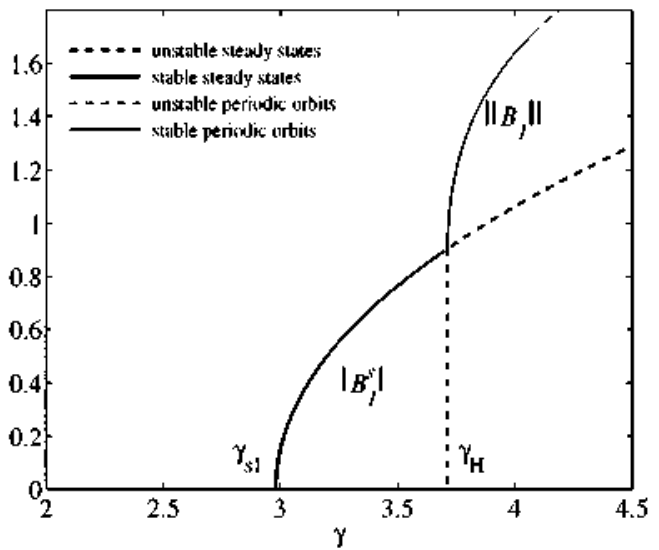

(e)

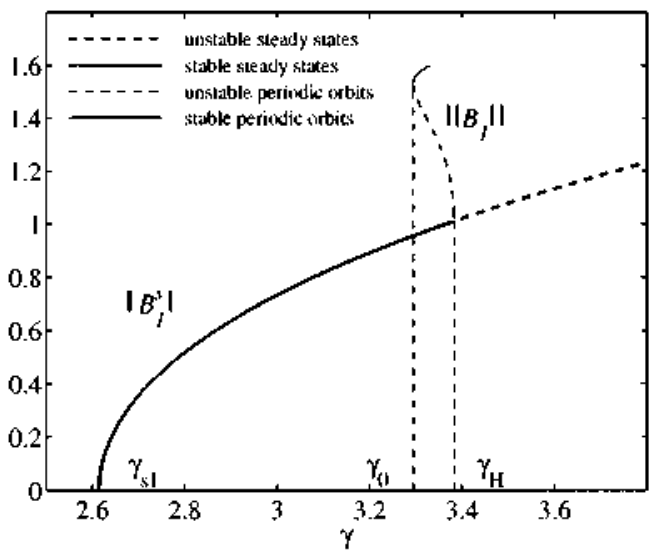

FIG. 3. (a) The critical values $\gamma_{s 1}, \gamma_{s 2}$, and $\gamma_{\mathrm{H}}$ in tems of $\tilde{\omega}$ for $\tilde{T}=$ -2.5 and $a=0.403$ (when only the eigenmode associated with $\Omega_{2}$ is djrectly excited). Notice that the system exhibits a unique, stable steady state in region $I$, two stable and one unstable steady state in region II, a stable and an unstable steady state in region III, and two unstable steady states in region IV. (b) $\left|B_{1}^{s}\right|$ and $\left|B_{2}^{s}\right|$ in terms of $\gamma$ for $\tilde{\omega}=0, \tilde{l}=-2.5$, and $a$ $=0.403$. (c) As in (b), but with $\tilde{\omega}=3.5, \tilde{I}=-2.5$, and $a=0.403$. (d) $\left|B_{1}^{s}\right|$ in tems of $\gamma$, and $\left\|B_{1}\right\|=\max \left\{\left|B_{1}(\tau)\right|: 0 \leqslant \tau \leqslant P\right\}$ for the branch of periodic solutions, of period $P$, that bifurcates at $\gamma=\gamma_{\mathrm{H}}$, for $\widetilde{\omega}=1.9368, \widetilde{l}=-2.5$, and $a=0.403$, (e) As in (c), but with $\bar{\omega}=2.195, \tilde{l}=-2.5$, and $a=0.403$.

$$
a\left|B_{1}\right|^{2}+\left|B_{2}\right|^{2}-\gamma\left(B_{2}+\overline{B_{2}}\right) / 2=0
$$

which is invariant under the symmetry (50); thus, symmetric attractors will lead to a symmetric set of intersections when plotting either the real or the imaginary part of $B_{1}$, and symmetry breaking and symmetry-gaining bifurcations will be clearly appreciated. That hypersurface has been selected be- 


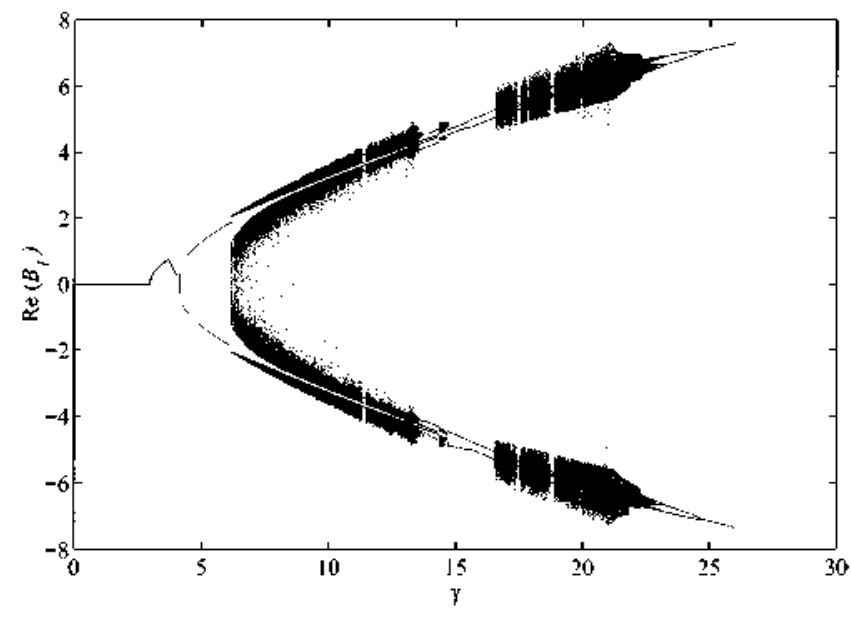

FIG. 4. Bifurcation diagram in terms of $\gamma$ for $\tilde{l}=-2.5$, $\tilde{\omega}=1.9368$, and $a$ $=0.403$. We are plotting the real part of $B_{1}$ for the intersection of the attractors with the Poincare hypersurface (58).

cause it contains the steady states and intersects the periodic orbits (at least twice) in each period: both properties readily follow from the equation

$$
\begin{aligned}
\frac{1}{2} \frac{d}{d \tau}\left(\left|B_{1}\right|^{2}+\left|B_{2}\right|^{2}\right)= & -\left(a\left|B_{1}\right|^{2}+\left|B_{2}\right|^{2}\right) \\
& +\gamma\left(B_{2}+\overline{B_{2}}\right) / 2,
\end{aligned}
$$

which is readily obtained when multiplying (46) by $\overline{B_{1}}$ and (47) by $\bar{B}_{2}$, adding and taking the real part. Equation (59) is. in fact, a first approximation of the mechanical energy equation: it gives a first approximation of the variation of the energy due to both viscous dissipation and forcing, and may be also used to show that the orbits of $(46)-(47)$ are bounded.

Periodic orbits exhibit a finite number of intersections on the Poincare hypersurface, and aperiodic orbits associated with chaotic attractors exhibit infinitely many intersections, which accumulate on a Cantor-set-like structure. In order to plot chaotic attractors, an appropriately large number of intersections will be plotted for the structure of the attractor to be somehow appreciated, after integrating (46)(47) in an appropriately large interval, to eliminate the initial transient when the orbit approaches the attractor. In order to ascertain chaotic dynamics, the first few Lyapunov exponents will be calculated as usually, ${ }^{29}$ by integrating (46)-(47) once and integrating the associated linearized problem for several. linearly independent initial conditions. and applying a Gram-Schmidt reorthonormalization procedure at $\tau$ $=T .2 T \ldots$, for some fixed $T$. Some unstable branches of periodic solutions [such as that in Fig. 3(e)] are calculated by means of a numerical continuation method. ${ }^{30}$

Now, the continuation of the bifurcation diagram in Fig. 3(d) for larger values of $\gamma$ is plotted in Fig. 4, where, for completeness, the steady states and periodic orbits in Fig. 3 (d) have been also included. The branch of nonsymmetric periodic orbits exhibits a period-doubling bifurcation at $\gamma$ $=\gamma_{1} \simeq 4.073$, and then several additional bifurcations (including a homoclinic symmetry gaining at $\gamma \simeq 4.095$, and a
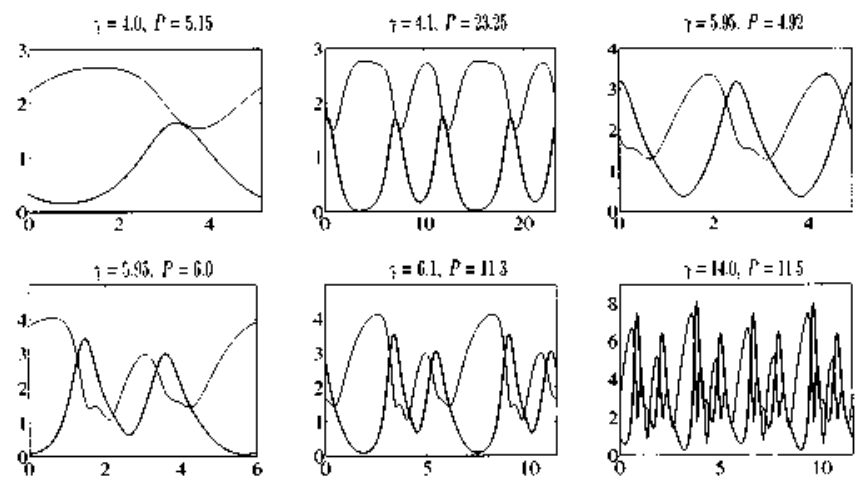

$\gamma=12.3, L=0.203$
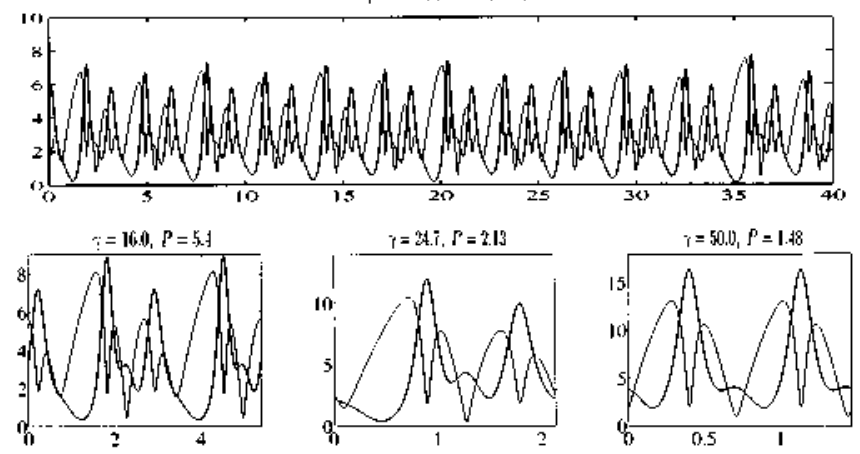

$\gamma=21.0, L=0.38$

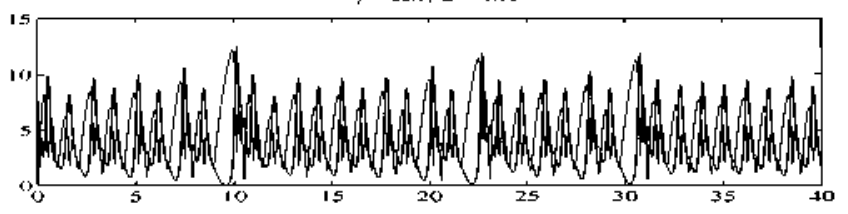

FIG. 5. $\left|B_{1}\right|$ (一) and $\left|B_{2}\right|$ (一) vs $\tau$ for several representative attractors in Fig. 4. The period $P$ and the first Lyapunov exponent $L$ are indicated for periodic and chaotic attractors, respectively. $\left(^{*}\right)$ The range of the parameter $y$ in which we expect quantitative agreement with experinents is described in remark (d) of See. V.

period-doubling sequence that accumulates at $\gamma \simeq 4.116$ ) in the interval $\gamma_{1}<\gamma<\gamma_{2} \simeq 4.15$, which is too narrow to be appreciated in Fig. 4. For $\gamma_{2}<\gamma<\gamma_{3} \simeq 6.16$ there is a branch of symmetric, periodic orbits that exhibits a supercritical pitchfork bifurcation at $\gamma=\gamma_{3}$. For $\gamma>\gamma_{3}$ the system jumps to a new symmetric, chaotic attractor (which alternates with periodic windows as $\gamma$ varies); if now $\gamma$ is decreased from $\gamma_{3}$, then the attractor exhibits several bifurcations and becomes unstable at $\gamma=\gamma_{4} \simeq 6$. Then the system exlibits hysteresis in the interval $\gamma_{4}<\gamma<\gamma_{3}$ (which again is too narrow to be appreciated in the figure). For $\gamma>\gamma_{3}$ the system gains and loses symmetry repeatedly. although only some of these transitions are clearly appreciated in Fig. 4 (for instance. the symmetry gaining at $\gamma=\gamma_{6} \simeq 15.55$ ). For illustration, some representative periodic and chaotic solutions are plotted in Fig. 5. where just one period is plotted for periodic solutions and a large time interval is plotted for chaotic ones in order to somelow appreciate irregularity: the period $P$ and the first Lyapunov $L$ exponent are indicated for periodic and chaotic solutions, respectively. Notice that the periodic solution at $\gamma=4$. Ishows a relaxation-like behavior because that value of $\gamma$ is quite close to the above-mentioned homoclinic bifurcation. Also, the periodic solutions in some periodic windows 


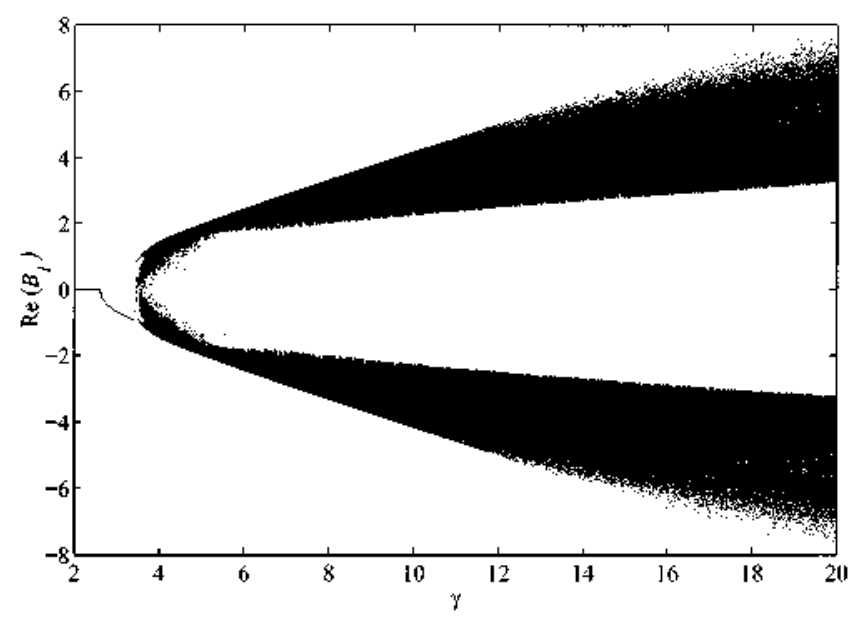

FIG. 6. The same as in Fig. 4, but with $\tilde{T}=-2.5, \widetilde{o}=2.1949$, and $a$ $=0.403$.

(e.g., that for $\gamma=14$ ) have a large period, and thus they exhibit a fairly complex behavior. Finally, notice that $\left|B_{1}\right|$ and $\left|B_{2}\right|$ are always essentially of the same size (even for $\gamma$ $=50$ : see Fig. 5) and thus none of the two involved modes dominates as $\gamma$ increases. as anticipated above.

The continuation of the bifurcation diagram in Fig. 3(e) is given in Fig. 6, and is fairly different from that in Fig. 4. For the sake of brevity. we just point out that as $\gamma$ $>\gamma_{\mathrm{H}} \simeq 3.358$ the system jumps to a new symmetric. periodic attractor that, after several bifurcations (not appreciated in Fig. 6) becomes chaotic (except for narrow periodic windows) through a period-doubling sequence. Notice that this attractor is not in the branch that bifurcated subcritically at $\gamma=\gamma_{\mathrm{H}}$ [see Fig. 3(e)] because the stable part of this branch disappeared at $\gamma \simeq 3.325<\gamma_{\mathrm{H}}$, and that the system exhibits hysteresis in $\gamma_{0}<\gamma<\gamma_{H}$ [also see Fig. 3(e)], but, again, this interval is fairly narrow and is not appreciated in Fig. 6. The periodic solutions for $\gamma<\gamma_{\mathrm{H}}$ are qualitatively similar to some of those plotted in Fig. 5, but the chaotic solutions for $\gamma>\gamma_{\mathrm{H}}$ exhibit a new, clearly (as $\gamma$ increases) intermittent behavior, as seen in Fig. 7. where the first Lyapunov exponent is indicated.

With the bifurcation diagrams described above. we just intended to illustrate the rich variety of transitions and large-
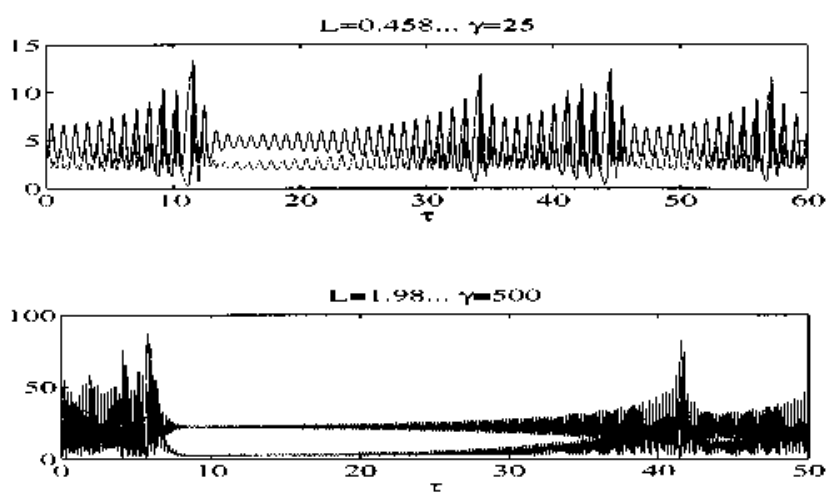

FIG. 7. $\left|B_{1}\right|$ (一) and $\left|B_{2}\right|$ (一) vs $\tau$ for two representative chaotic attractors in Fig. 6. The first Lyapunov exponent $L$ is indicated. [See the ${ }^{*}$ ) in the caption of Fig. 5.] time behaviors of the system. The analysis above and that of several additional bifurcation diagrams not presented here for the sake of brevity allows us to draw the following conclusions concerning the dynamics of the liquid bridge near the $2: 1$ resonance at $\Lambda_{0} \simeq 2.23$ (similar conclusions apply near the other resonance), when only the mode associated with the higher frequency is directly excited.

(a) If either (55) does not hold or it holds but $\widetilde{\omega}$ is outside the interval $\left[\widetilde{\omega}_{2}^{-}, \widetilde{\omega}_{2}^{+}\right]$, then the bifurcation diagram for (varying values of the scaled forcing amplitude $\gamma$ ) is as that in Fig. 3(b) if $\widetilde{\omega}_{1}^{-}<\widetilde{\omega}<\widetilde{\omega}_{1}^{+}$, and as that in Fig. 3(c) otherwise. As $\gamma$ increases the mode with lower frequency (which is not directly excited) dominates.

(b) If, instead, (55) holds and $\widetilde{\omega_{2}}<\widetilde{\omega}<\widetilde{\omega}_{2}^{+}$, then, as $\gamma$ increases, the system also exhibits first a Hopf bifurcation and then additional bifurcations leading to quite a rich variety of complex dynamics, including bichromatic oscillations with periodic or chaotic modulation. Now, none of the two involved modes dominates as $\gamma$ increases.

\section{Forcing with the lower frequency $\Omega_{1}\left(\beta_{2}^{+}=\beta_{2}^{-}=0\right)$}

Since the analysis is similar to that in the last section. some details will be omitted. The constant $\omega$ is now selected as

$$
\omega=\omega_{1} .
$$

to rewrite Eqs. (40) and (4I) as

$$
\begin{aligned}
& d B_{1} / d \tau=-(a+i \tilde{\omega}) B_{1}+i \overline{B_{1}} B_{2}+\gamma, \\
& d B_{2} / d \tau=-[1+2 i(\widetilde{\omega}+\tilde{l})] B_{2}+i B_{1}^{2},
\end{aligned}
$$

where the scaled forcing amplitude $\gamma$ is now given by

$$
\gamma=i\left(\beta_{1}^{+}+\beta_{1}^{-}\right),
$$

and will again be selected to be real and positive. Also, if both disks are vibrated then they must not be vibrated in counterphase with the same amplitude (otherwise $\gamma$ identically vanishes). The normal form (61)-(62) is again invariant under the symmetry (49), and thus when changing simultaneously the signs of $\widetilde{l}$ and $\widetilde{\omega}$ the dynamics of (61)-(62) stands. The steady states are readily seen to be given by

$$
\begin{aligned}
& \left|B_{1}^{s}\right|^{6}+2[a-2 \widetilde{\omega}(\widetilde{\omega}+\tilde{l})]\left|B_{1}^{s}\right|^{4}+\left[\left(a^{2}+\widetilde{\omega}^{2}\right)\left|B_{1}^{s}\right|^{2}-\gamma^{2}\right] \\
& \quad \times\left[1+4(\widetilde{\omega}+\widetilde{l})^{2}\right]=0, \\
& \left|B_{2}^{s}\right|^{2}=\left|B_{1}^{s}\right|^{4} /\left[1+4\left(\widetilde{\omega}+\widetilde{l}^{2}\right],\right.
\end{aligned}
$$

and correspond to bichromatic oscillations of the liquid bridge. Their multiplicity and stability properties are analyzed by means of Eqs. (B5)-(B6) in the Appendix. Equation (B5) may be written as

$$
\begin{aligned}
3\left|B_{1}^{s}\right|^{4}+4[a-2 \widetilde{\omega}(\widetilde{\omega}+\widetilde{l})]\left|B_{1}^{s}\right|^{2}+\left(a^{2}+\widetilde{\omega}^{2}\right) \\
\times\left[\mathbf{1}+\mathbf{4}(\widetilde{\omega}+\widetilde{l})^{2}\right]=0 .
\end{aligned}
$$

When requiring Eqs. (63) and (65) to possess a common solution $\left|B_{1}^{s}\right| \geqslant 0$, we obtain a fairly involved equation that is omitted and always yields two cusped curves, as the ones plotted in Figs. 8(a) and 9(a): those two cusped curves give 
the limits of the multiplicity interval. $\gamma_{s 1}<\gamma<\gamma_{s 2}$, where the system exlibits three steady states. The cusp points of these curves occur at $\widetilde{\omega}=\widetilde{\omega}_{1}^{-}$and $\widetilde{\omega}=\widetilde{\omega}_{1}^{+}$, where $\widetilde{\omega}_{1}^{ \pm}$are the real solutions of

$$
0<2[\widetilde{\omega}(\widetilde{\omega}+\tilde{l})-a]=\left\{3\left(a^{2}+\widetilde{\omega}^{2}\right)\left[1+4\left(\widetilde{\omega}+\tilde{l}^{2}\right]\right\}^{1 / 2} .\right.
$$

Condition (B6) (giving the Hopf bifurcation points) leads to a quite involved equation that must be solved numerically: we only can see analytically that the left-hand side of (B6) is positive for small forcing, $0<\gamma \ll 1$, and negative if $\gamma$ is sufficiently large; in addition, the left-hand side of (B6) is seen to be positive on the cusped curves associated with condition (B5). Thus, a Hopf bifurcation point must exist for at least one value of $\gamma_{,} \gamma_{\mathrm{H}}$, if $\tilde{l}$ and $\widetilde{\omega}$ are kept fixed, and $\gamma_{\mathrm{H}}$ is always bigger than the multiplicity limits $\gamma_{s 1}$ and $\gamma_{s 2}$, as in the plots in Figs. 8(a) and 9(a). Notice that the plot in Fig. 8 (a) is symmetric in $\tilde{\omega}$ [as a consequence of the invariance of (61)-(62) under the symmetry (49)]. This symmetric case will be considered below in some detail because (unlike its counterpart in Sec. IV A I) a Hopf bifurcation (and subsequently a more complex chaotic dynamics) appears for sufficiently large $y$. In addition. we shall consider the nonsymmetric case in Fig. 9(a).

Now, for fixed values of the detuning parameters $\widetilde{\omega}$ and $\tilde{l}$, the plot of the steady states in terms of the scaled forcing parameter $y$ is always either monotonous or $\mathrm{S}$ shaped. as in Figs. 8(b) and 8(c) depending on whether $\widetilde{\omega}_{1} \leqslant \widetilde{\omega} \leqslant \widetilde{\omega}_{1}^{+}$or not. Also. the stability criteria in Appendix B shows that when the plot is monotonous the steady states are asy mptotically stable if $y<\gamma_{\mathrm{H}}$ (where $\gamma_{\mathrm{H}}$ corresponds to the Hopf bifurcation point mentioned above), as indicated in Figs. 8(b). 9(b): when the response curve is $S$ shaped. as in Fig. 8 (c), the lower branch and the part of the upper branch for $y<\gamma_{\mathrm{H}}$ correspond to asymptotically stable steady states, while the intermediate branch and the part of the upper branch for $\gamma>\gamma_{\mathrm{H}}$ correspond to unstable steady states. For completion, a part of the branch of periodic orbits that bifurcates at $\gamma=\gamma_{\mathrm{H}}$ has also been plotted in Figs. 8(b) and 9(b), as obtained by numerical continuation. Notice that as $\gamma$ grows, $\left|B_{2}^{s}\right| /\left|B_{1}^{s}\right|$ becomes quite large, and thus the mode corresponding to $\Omega_{2}=2 \Omega_{1}$ (which is not directly excited) dominates the directly excited mode (corresponding to $\Omega_{1}$ ). as in Sec. IV A 1; this was to be expected because, according to $(63)-(64) .\left|B_{2}^{s}\right| \sim\left|B_{1}^{s}\right|^{2} \rightarrow \infty$, as $\gamma \rightarrow \infty$. But the steady states are unstable for sufficiently large $\gamma$ (i.e., for $\gamma>\gamma_{\mathrm{H}}$ ) and, as in Sec. IB A 1, in the more complex (periodic or chaotic) attractors that appear for large $\gamma$, none of the two involved modes dominates the other one [as suggested in Figs. 8(b) and 9(b), 11, and 13]

As in Sec. IV A 1, in order to plot the attractors for higher values of $\gamma$, we shall consider a Poincare map. To this end, we shall plot (the real part of $B_{1}$ for) the intersections (pointing outward) of the periodic or chaotic attractors with the Poincare hyperellipsoid.

$$
a\left|B_{1}\right|^{2}+\left|B_{2}\right|^{2}-\gamma\left(B_{1}+\overline{B_{1}}\right) / 2=0,
$$

which, again, contains the steady states and intersects the periodic orbits in each period. (a)

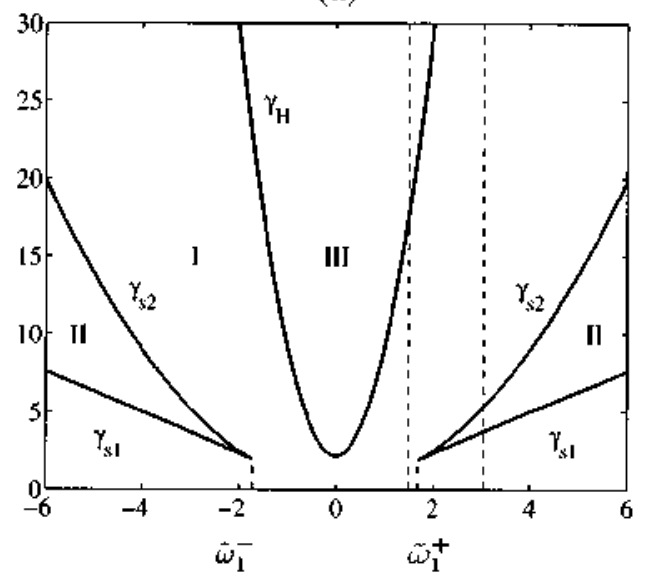

(b)

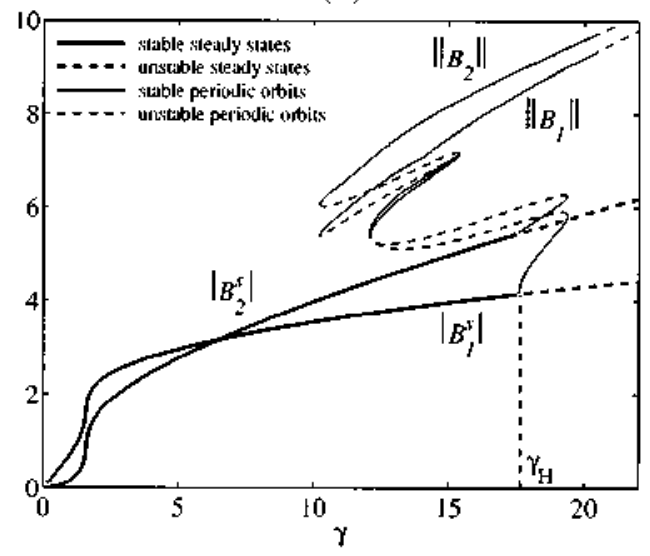

(c)

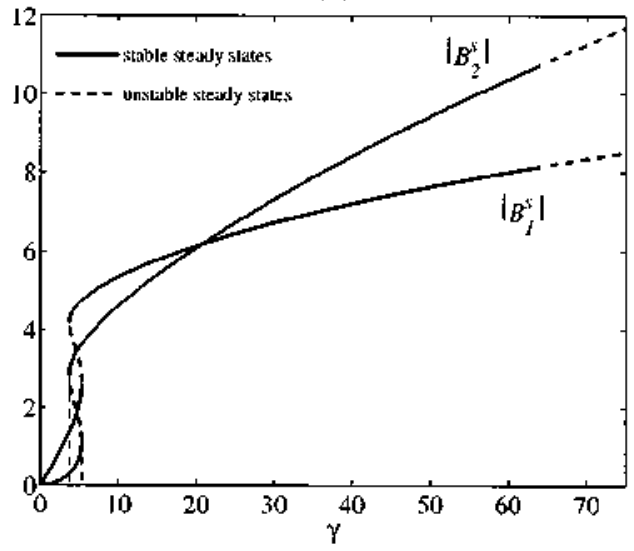

FIG, 8. (a) The critical values $\gamma_{s 1}, \gamma_{s 2}$, and $\gamma_{\mathrm{H}}$ in terms of $\sigma$ for $\tilde{t}=0$ and $a=0.403$ (when only the eigennode associated with $\Omega_{1}$ is directly excited). The system exlibits a unique, stable steady state in region $\mathrm{I}$, two stable and one unstable steady states in region II, and one unstable steady state in region III. (b) $\left|B_{1}^{s}\right|,\left|B_{2}^{s}\right|,\left\|B_{1}\right\|$, and $\left\|B_{2}\right\|$ in terms of $\gamma$, with the norm $\|+\|$ as defined in Fig. 3(d), for the branch of periodic orbits that bifurcates at $y$ $=\gamma_{\mathrm{H}}$. for $\sigma=1.5, \tilde{i}=0$, and $a=0.403$. (c) $\left|B_{1}^{s}\right|$ and $\left|B_{2}^{s}\right|$ vs $\gamma$ for $\sigma$ $=3.05, \tilde{l}=0, a=0.403$. [See the (*) in the caption of Fig. 5.]

Let us first consider, in Fig. 10, the continuation of the bifurcation diagram in Fig. 8(b). Notice that the latter exhibits four bending points, at $\gamma_{1} \simeq 19.5, \gamma_{2} \simeq 12, \gamma_{3} \simeq 16$, and $\gamma_{4}=10$, the solution changing from stable to unstable when 
(a)

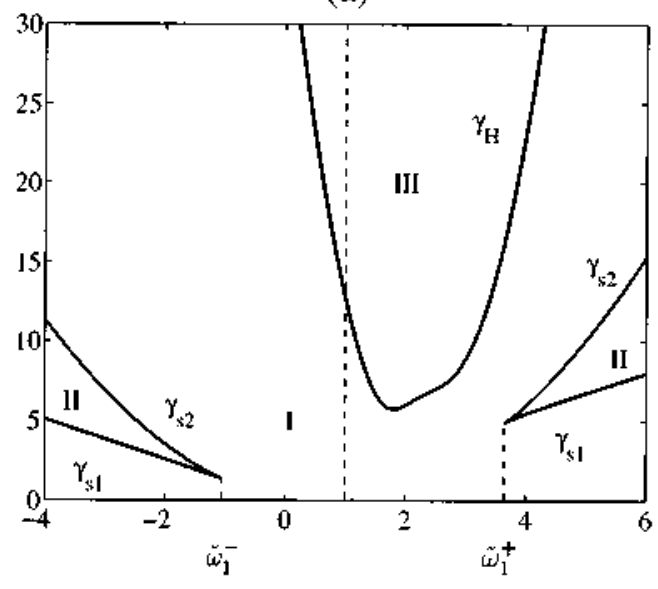

(b)

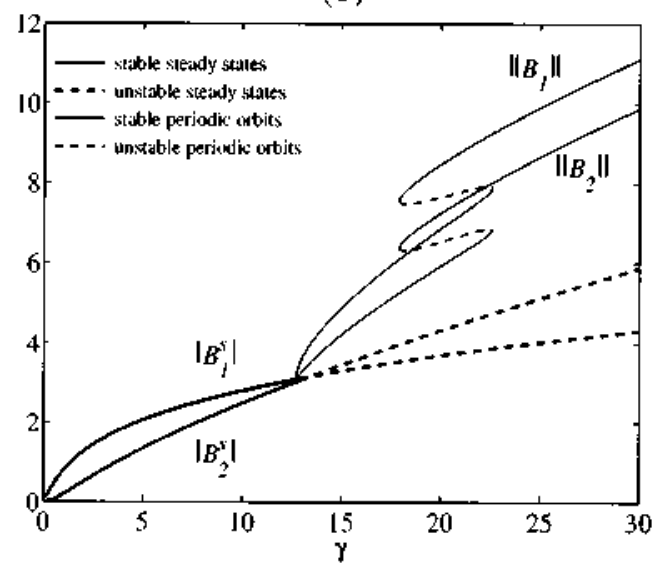

FIG. 9. (a) The sane as in Fig. 8(a), but with $T=-2.5$ and $a=0.403$. (b) As in Fig. 8(b), but with $\tilde{\omega}=1, \tilde{T}=-2.5$, and $a=0.403$.

going up through each bending point: thus, the system exhibits hysteresis in the interval $\gamma_{4}<\gamma<\gamma_{1}$, with three possible stable states (one of them steady and the other two periodic) in the subinterval $\gamma_{2}<y<\gamma_{3}$. For the sake of clarity we have plotted again in Fig. 10 a part of the branch of periodic solutions in Fig. 8(b). Also, in order to obtain a not-too-involved plot in Fig. 10, the bifurcation parameter $\gamma$ is only moved upward and thus some intervals where hysteresis appears between two different attractors are not appreciated (hysteresis would be seen if $\gamma$ were also allowed to move downward). Notice that at $\gamma=\gamma_{1}=19.5$ the system jumps to a new periodic attractor [as was to be expected: see Fig. 8(b)] that shows three intersections with the Poincaré hypersurface (while the periodic attractor in $18 \approx \gamma<\gamma_{1}$ only exhibited one intersection): this is so just because the geometric shape of the closed orbit associated with the new periodic attractor in the phase space is more involved than that associated with the former attractor (similarly, new branches appear at $\gamma \simeq 25$ and $\gamma \simeq 29.5$ that do not correspond to actual bifurcations, but they just correspond to new intersections of the periodic orbit with the Poincare hypersurface). The new branch that appears at $\gamma=\gamma_{1}$ exhibits a first period doubling at $\gamma=\gamma_{5}=20.2$ and then a period-doubling sequence that

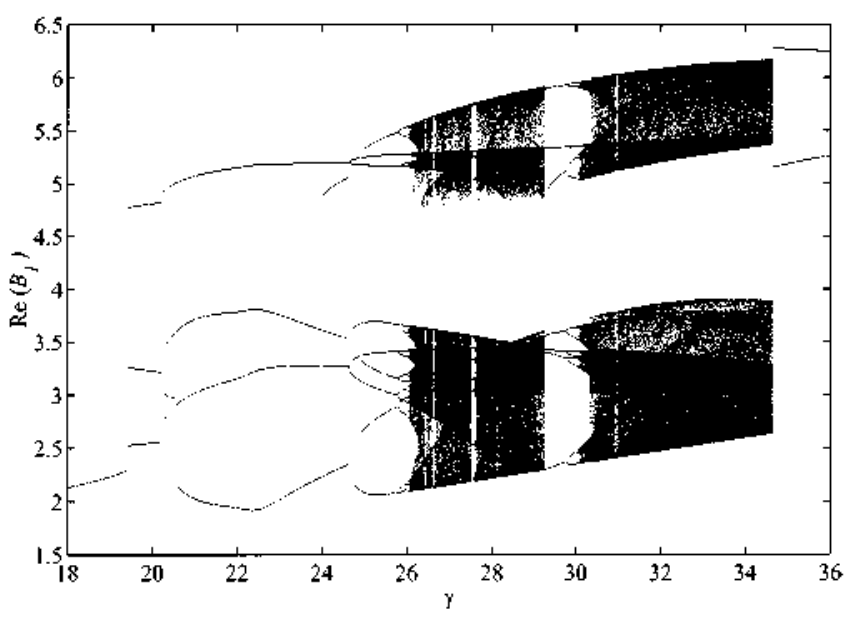

FIG. 10. Bifurcation diagram in terms of $\gamma$ for $l=0, \tilde{\omega}=1.5$, and $a$ $=0.403$. The real part of $B_{1}$ is plotted for the intersections of the attractors with the Poincare hypersurface (65). [See the (*) in the caption of Fig. 5.]

accumulates at $\gamma=\gamma_{6}=26.3$. The resulting chaotic attractor alternates with periodic windows as usually and loses stability at $\gamma=\gamma_{7}=34.5$. where the system jumps to a new periodic attractor that exhibits two intersections with the Poincare hypersurface. Notice that the periodic windows exhibit intermittency (of type I in our case) at one end and a perioddoubling sequence at the other end, as is frequently the case in simpler dynamical systems. ${ }^{31}$

For illustration, some representative periodic and chaotic solutions associated with attractors in Figs. 8(b) and 10 are plotted in Fig. 11, where just one period is plotted for periodic solutions and the first Lyapunov exponent is indicated for chaotic ones. Notice that one of the periodic solutions at $\gamma=17.78$ (precisely the one that is closer to the unstable steady state) exhibits much higher values of $\left|B_{2}\right|$ than those of $\left|B_{1}\right|$ (as it occurred with the steady states for large $y$ ), but the other one shows similar values of both complex amplitudes, and the same is tne for the remaining solutions plotted in Fig. 11, even for $\gamma$ as large as 140; thus both involved modes show a similar activity for large $\gamma_{\text {. as anticipated }}$ above. Again, as in Sec. IV A 1, some of the periodic solutions with a large period exhibit a quite complex behavior and are not quite different from the chaotic ones at first sight (compare the solutions for $\gamma=25.5$ and $\gamma=32$ ).

The bifurcation diagram for $\tilde{l}=0, \tilde{\omega}=1.5$ and the associated attractors in Figs. 8(b). 10, and 11 are representative of those for other values of $\tilde{l}$ and $\tilde{\omega}$. See the continuation of the bifurcation diagram in Fig. 9(b) and some representative periodic and chaotic attractors, which are given in Figs. 12 and 13. In fact, the appearance of involved bifurcation diagrams is the rule, but there are exceptions to this rule: for instance, for $\tilde{l}=\tilde{\omega}=0$, the periodic branch that bifurcates from the steady-state branch at $\gamma=\gamma_{\mathrm{H}}$, remains stable for all $\gamma>\gamma_{\mathrm{H}}$, and the system shows no transitions to chaotic attractors.

\section{B. Cubic nonlinearity ( $\Omega_{2}$ associated with an odd mode)}

In this case the coefficients, $\alpha_{21}$ and $\alpha_{22}$, of the quadratic, nonlinear terms vanish in the amplitude equations 

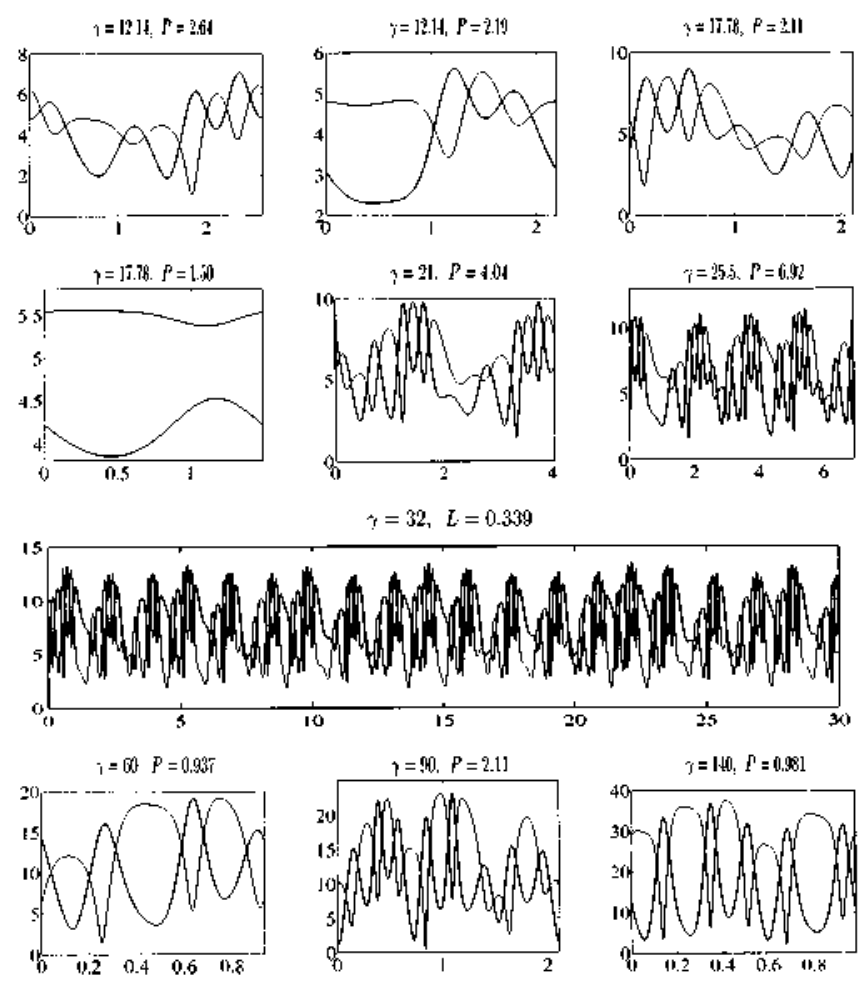

$\hat{t}=32, L=0.339$

FIG. 11. $\left|B_{1}\right|(-)$ and $\left|B_{2}\right|$ (-) vs $\tau$ for several representative attractors in Fig. 10. The period $P$ and the first Lyapunov exponent $L$ are indicated for periodic and chaotic attractors respectively. [See the $\left({ }^{*}\right)$ in the caption of Fig. 5.]

(18), according to remark (c) at the end of Sec. III. Then we must consider saturating cubic terms in the amplitude equations, which are still those in Eq. (18), with

$N_{k}=i \epsilon\left(\alpha_{2 k 1}\left|A_{1}\right|^{2}+\alpha_{2 k 2}\left|A_{2}\right|^{2}\right) A_{k}$, for $k=1$ and 2 ,

where $\alpha_{2 k 1}$ and $\alpha_{2 k 2}$ are seen to be real and (generically) nonzero. In fact, $\alpha_{211}$ and $\alpha_{222}$ were calculated in Ref. 19 and the other two coefficients may be calculated by a similar (and somewhat tedious) procedure. Notice that the same cubic nonlinear terms would be obtained if $\Omega_{2} \neq 2 \Omega_{1}$; the $2: 1$

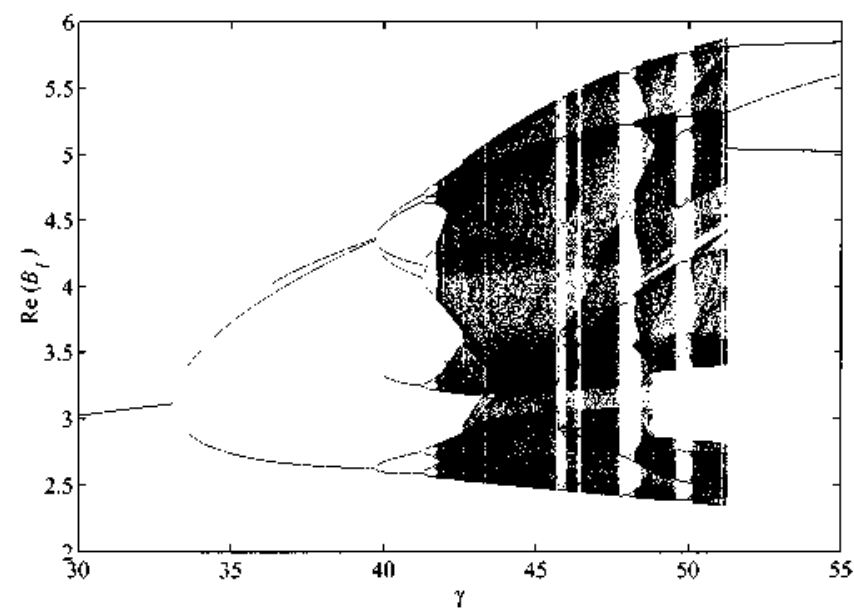

FIG. 12. The same as in Fig. 10, but with $\tilde{\tau}=-2.5, \tilde{\omega}=1$, and $a=0.403$. [See the (*) in the caption of Fig. 5.]
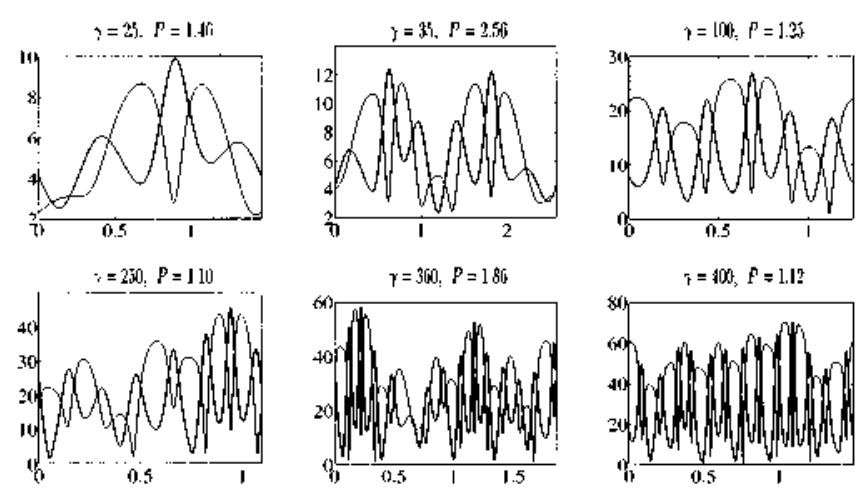

$=1 \mathrm{j}, L=0,291$

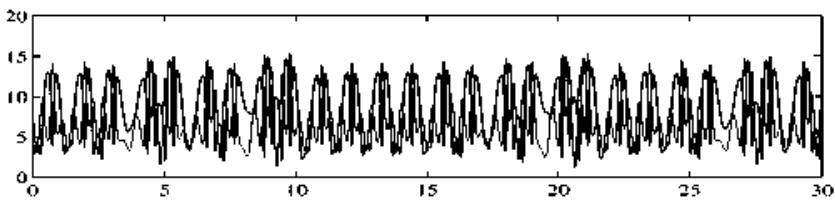

$\gamma=13 i, L=0.4(i \pi$

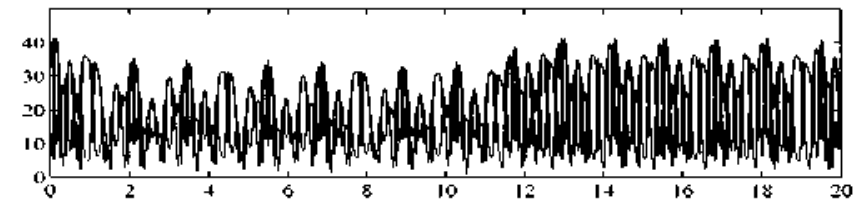

FIG. 13. The same as in Fig. 11 for several representative attractors in Fig. 12. [See the (*) in the caption of Fig. 5.]

resonance appears now at fourth order but the effect of quartic terms may be neglected in the first approximation (if the coefficients of cubic terms are nonzero). Now, if only one mode is directly excited, that is, if either $\beta_{1}^{+}=\beta_{1}^{-}=0$ or $\beta_{2}^{+}=\beta_{2}^{-}=0$ in $(10)$ and $(18)$, then for $k=1$ or 2 we have

$$
\delta d\left|A_{k}\right|^{2} / d \tau=-\left(\alpha_{4 k} \sqrt{C}+\alpha_{5 k} C\right)\left|A_{k}\right|^{2},
$$

as readily obtained when neglecting HOT in (18), multiplying the resulting equation by $\overline{A_{k}}$ and adding the complex conjugate. Thus the not-directly-excited mode decays exponentially in the viscous time scale $t \sim\left|\alpha_{4 k} \sqrt{C}+\alpha_{5 k} C\right|$ (which is the slow scale for the evolution of the modulation of the excited mode). If. consequently. we neglect the notexcited mode, the evolution of the other mode coincides with that analyzed in Ref. 19. where it was seen that the associated dynamics is fairly simple

\section{CONCLUDING REMARKS}

We have analyzed the $2: 1$ internal resonances that appear in liquid bridges in the interval $0<\Lambda<\pi$. To this end. we first derived in Sec. III a normal form giving the slow evolution of the complex amplitudes of the two involved modes. We have seen that only the resonances at $\Lambda \simeq 0.249$ and $\Lambda \simeq 2.23$ lead to nonzero quadratic nonlinearities in the normal form. which are responsible for resonant coupling between the two modes (which in tum is the essential mechanism that leads to complex dynamics when this resonance comes into play): the remaining 2:1 resonances lead to the usual cubic nonlinearities and the associated weakly nonlinear dynamics is fairly simple, as seen in Sec. IV B. The quadratic normal form has been analyzed in Sec. IV A, 
where numerical applications have been made to the internal resonance at $\Lambda \simeq 2.23$. We have analyzed the cases when the forcing frequency is close to either $\Omega_{1}$ and $\Omega_{2} \simeq 2 \Omega_{1}$. by first calculating the steady states of the normal form (corresponding to purely mono- and bichromatic oscillations of the liquid bridge) and their linear stability. In addition, several bifurcation diagrams have been analyzed in some detail to illustrate the wealth of transitions and large-time dynamics: the latter include periodic and chaotic solutions that correspond to slowly modulated. bichromatic oscillations of the liquid bridge. Some remarks are now in order.

(a) The not-directly-excited mode usually dominates in the steady states of the normal form as the forcing amplitude increases. In periodic and chaotic behaviors, instead, both modes exhibit a similar activity for large forcing.

(b) The transition from regular. steady or periodic solutions, to irregular, chaotic ones, can be somewhat smooth, through a periodic-doubling sequence, as in Fig. 10. but it can be also discontinuous, as in Fig. 6. Still, the system exhibits multiple attractors in some parameter ranges that lead to hysteresis when the forcing amplitude is slowly varied.

(c) The bifurcation diagrams have been plotted in terms of the scaled forcing amplitude $\gamma$. If. instead, $\gamma$ and the detuning parameter $\tilde{l}$ [which is a scaled measure of how close to the 2:1 resonance we are. and depends only on the slenderness $\Lambda$ once the liquid has been chosen. according to (13). (38), and (43)] are kept fixed, and the detuning parameter $\tilde{\omega}$ [a scaled measure of how close the forcing frequency is to either $\Omega_{1}$ or $\Omega_{2}=2 \Omega_{1}$, according to (10), (38), (43). and either (45) or (60)] is varied, then we move along a horizontal straight line on the diagrams in Figs. 3(a), 8(a), and 9(a). Our discussion in Sec. IV A readily implies that the possible periodic or chaotic behavior appear only in a finite interval. $\widetilde{\omega}_{0}^{-}<\widetilde{\omega}<\widetilde{\omega}_{0}^{+}$, while outside this interval (which can be void) the solution always converges to a steady state.

(d) Since we have neglected gravity, our results above must be used with care when comparing with experiments on Earth. If, for illustration, water is used and the radius of the bridge is $1 \mathrm{~mm}$, then the Bond and capillary numbers are $B$ $\simeq 0.14$ and $C \simeq 4 \times 10^{-3}$ : then $\epsilon \simeq 0.26, \mu \simeq 0.03$, and $b$ $\simeq 2.64$ [see Eqs. (37), (38). and (42)]. and the requirements $\mu\left|\beta^{ \pm}\right|=\gamma \mu / b \ll 1$ or $\mu\left|\beta^{ \pm}\right|=\gamma \mu \ll 1$ (depending on whether we are forcing with the higher or the lower frequency, as in Secs. IV A 1 and IV A 2, respectively) allow a not-too-large value of the scaled forcing parameter $\gamma$, say $\gamma$ $\leqslant 15$ and $\gamma \leqslant 6$, respectively. Thus only the left part of the bifurcation diagrams in Figs. 3(b)-3(c). 4, 6, 8(b)-8(c), and 9 (b), and the first few plots in Fig. 5, apply for quantitative comparison with experiments on Earth; notice that $R$ cannot be significantly increased because $B$ must be small. Notice also that, if either $\gamma$ is increased or the experimental conditions are varied (in such a way that either $B$ or $C$ are no longer small), then our results could still apply qualitatively. In any case, they show that chaotic behavior is likely to be experimentally observed near the second-order resonances (which could be determined experimentally if the Bond number is not small). For experiments in sounding rockets or space platforms, instead, the quantitative scope of our results is much wider. In this case, $B$ is small for a larger radius, say, $R=5 \mathrm{~cm}$. Then $C \simeq 6 \times 10^{-4}, \epsilon \simeq 0.04, \mu \simeq 6 \times 10^{-4}$, and $\gamma$ can be as large as, say, 750 and 300 (in the two cases considered above); thus, in this case, all results in the paper are expected to apply for quantitative comparison with experiments.

\section{ACKNOWLEDGMENTS}

This research was supported by DGICYT and NASA under Grants No. PB96-0130 and No. 96-H01-115.

\section{APPENDIX A: SOME USEFUL EXPRESSIONS}

Here we collect several groups of involved algebraic expressions that were omitted in Sec. III to facilitate the reading of that section.

The first group deals with the solution of the linear eigenvalue problem $(20)-(25)$, which was first solved in a semianalytical form by Sanz. ${ }^{13}$ We simply collect his results here. The eigenfrequencies, $\Omega_{k}$, are the real solutions of one of the following equations:

$$
\Lambda_{0} \tan \Lambda_{0}=\sum_{n \text { odd }} a_{k n^{r_{n}}}
$$

or

$$
\Lambda_{0} \cot \Lambda_{0}+\sum_{n \text { even }} a_{k n} r_{n}=0
$$

where

$$
\begin{aligned}
& a_{k 0}=1, \quad a_{k n}=2 \Omega_{k}^{2} /\left(\Omega_{k}^{2} q_{n}-s_{n}\right), \quad \text { if } n \geqslant 1, \\
& q_{n}=I_{0}\left(l_{n}\right), \quad r_{n}=q_{n} /\left(l_{n}^{2}-1\right), \quad s_{n}=l_{n}\left(l_{n}^{2}-1\right) I_{1}\left(l_{n}\right) \\
& l_{n}=n \pi /\left(2 \Lambda_{0}\right) . \quad \text { if } n \geqslant 0 ;
\end{aligned}
$$

here $I_{i}$ and $I_{1}$ are the first two modified Bessel functions. If the first equation (Al) holds (odd modes), then $Q_{k}$ and $F_{k}$ are given by

$$
\begin{aligned}
Q_{k}= & \sum_{n \text { odd }} a_{k n} I_{0}\left(l_{n} r\right) \cos \left[I_{n}\left(z+\Lambda_{0}\right)\right], \\
F_{k}= & \Lambda_{0}\left(\cos \Lambda_{0}\right)^{-1} \sin z+\sum_{n \text { oild }} a_{k n n} r_{n} \\
& \times \cos \left[l_{n}\left(z+\Lambda_{0}\right)\right],
\end{aligned}
$$

up to a common complex factor, while $U_{k}$ and $W_{k}$ may be calculated from (A3) as [see (21)]

$$
U_{k}=i Q_{k r} / \Omega_{k}, \quad W_{k}=i Q_{k z} / \Omega_{k} .
$$

If instead the second equation (A1) holds (even modes) then 


$$
\begin{aligned}
Q_{k}= & \sum_{n \text { even }} a_{k n} I_{0}\left(l_{n} r\right) \cos \left[I_{n}\left(z+\Lambda_{0}\right)\right], \\
F_{k}= & \Lambda_{0}\left(\sin \Lambda_{0}\right)^{-1} \cos z+\sum_{n \text { even }} a_{k n} r_{n} \\
& \times \cos \left[l_{n}\left(z+\Lambda_{0}\right)\right] .
\end{aligned}
$$

In the second group of expressions we give the (real) coefficients $\alpha_{4 k}$ and $\alpha_{5 k}$ of the amplitude equations (18). as taken from Ref. 19.

$$
\begin{aligned}
& \alpha_{4 k}= \int_{0}^{1} Q_{k r}\left(r, \Lambda_{0}\right)^{2} r d r\left(\sqrt{2 \Omega_{k}^{3}} \int_{-\Lambda_{0}}^{\Lambda_{0}}\left(F_{k}^{\prime 2}-F_{k}^{2}\right) d z\right)^{-1} \\
&\left(\alpha_{5 k}-2-2 \alpha_{4 k}^{2} / \Omega_{k}\right) \int_{-\Lambda_{0}}^{\Lambda_{0}}\left(F_{k}^{\prime 2}-F_{k}^{2}\right) d z \\
&=-4 F_{k}^{\prime}\left(\Lambda_{0}\right) F_{k}^{\prime \prime}\left(\Lambda_{0}\right) \\
&-\int_{0}^{1} Q_{k r}\left(r, \Lambda_{0}\right) Q_{k r}^{*}\left(r . \Lambda_{0}\right) r d r / \sqrt{2 \Omega_{k}^{3}} \\
&+\int_{-\Lambda_{0}}^{\Lambda_{0}}\left[2 Q_{k}(1, z)^{2}-\Omega_{k}^{2} F_{k}(z)^{2}\right. \\
&\left.+\alpha_{4 k} O_{k}(1, z) F_{k}^{*}(z)\right] d z
\end{aligned}
$$

where $Q_{k}$ and $F_{k}$ are as given by (A3) or (A3 $)$, depending on whether $\Omega_{k}$ satisfies the first or the second equation (A1), and $Q_{k}^{*}$ and $F_{k}^{*}$ are as given by

$$
\begin{aligned}
\sqrt{2 \Omega_{k}} Q_{k}^{*}= & b Q_{k}+\partial Q_{k} / \partial \Lambda_{0}+\sum_{n \text { odd }} b_{k n} I_{0}\left(l_{n} r\right) \\
& \times \cos \left[I_{n}\left(z+\Lambda_{0}\right)\right], \\
\sqrt{2 \Omega_{k}} F_{k}^{*}= & b F_{k}+\partial F_{k} / \partial \Lambda_{0}+2 \Lambda_{0}\left(\partial \Omega_{k} / \partial \Lambda_{0}\right. \\
& \left.-\alpha_{4 k} \sqrt{2 \Omega_{k}}\right) \sin z /\left(\Omega_{k} \cos \Lambda_{0}\right) \\
& +\sum_{n \text { odkl }} b_{k n} r_{n} \cos \left[l_{n}\left(z+\Lambda_{0}\right)\right] .
\end{aligned}
$$

if $\Omega_{k}$ satisfies the first equation (A1), while if $\Omega_{k}$ satisfies the second equation (A1), then $Q_{k}^{*}$ and $F_{k}^{*}$ are

$$
\begin{aligned}
\sqrt{2 \Omega_{k}} Q_{k}^{*}= & b Q_{k}+\partial Q_{k} / \partial \Lambda_{0}+\sum_{n \text { even }} b_{k n} I_{0}\left(l_{n} r\right) \\
& \times \cos \left[I_{n}\left(z+\Lambda_{0}\right)\right], \\
\sqrt{2 \Omega_{k}} F_{k}^{*}= & b F_{k}+\partial F_{k} / \partial \Lambda_{0}+2 \Lambda_{0}\left(\partial \Omega_{k} / \partial \Lambda_{0}\right. \\
& \left.-\alpha_{4 k} \sqrt{2 \Omega_{k}}\right) \cos z /\left(\Omega_{k} \sin \Lambda_{0}\right) \\
& +\sum_{n \text { even }} b_{k n} r_{n} \cos \left[I_{n}\left(z+\Lambda_{0}\right)\right] .
\end{aligned}
$$

In (A7) $-\left(\mathrm{A} 7^{\prime}\right) . b$ is an arbitrary constant,

$$
b_{k n}=2 \Omega_{k}\left(\partial \Omega_{k} / \partial \Lambda_{0}-\sqrt{2 \Omega_{k}} \alpha_{4 k}\right) q_{n} a_{k n} /\left(\Omega_{k}^{2} q_{n}-s_{n}\right),
$$

and $a_{k n}, l_{n}, q_{n}, r_{n}$, and $s_{n}$ are as given in (A2).
In the third group of expressions we give the forcing terms in Eqs. (26) $-(31)$, which are

$$
\begin{aligned}
& g_{1}^{1}=i \sum_{k=1,2}\left(\alpha_{1 k}^{+} \beta_{k}^{+}-\alpha_{1 k}^{-} \beta_{k}^{-}\right) U_{k} e^{j\left(\Omega_{k^{t}}+\omega_{k} \tau\right)}+\text { c.c., } \\
& g_{1}^{2}=i \sum_{k=1,2}\left(\alpha_{1 k}^{+} \beta_{k}^{+}-\alpha_{1 k}^{-} \beta_{k}^{-}\right) W_{k} e^{l\left(\Omega_{k^{t}} t \omega_{k} \tau\right)}+\text { c.c., } \\
& G_{1}^{ \pm}=i \sum_{k=1.2} \Omega_{k} \beta_{k}^{ \pm} e^{i\left(\Omega_{k} t+\phi_{k} \tau\right)}+\text { c.c., } \quad L_{1}^{ \pm}=0, \\
& H_{1}^{1}=i \sum_{k=1,2}\left(\alpha_{1 k}^{+} \beta_{k}^{+}-\alpha_{1 k}^{-} \beta_{k}^{-}\right) F_{k} e^{j\left(\Omega_{k^{t}} t \omega_{k} \tau\right)}+\text { c.c., }
\end{aligned}
$$

$$
H_{1}^{2}=0, \quad 2 M_{1}=\sum_{k=1,2}\left(\beta_{k}^{+}-\beta_{k}^{-}\right) e^{i\left(\Omega_{k} t+\omega_{k} t\right)}+\text { c.c. } .
$$$$
g_{2}^{l}=i\left(\alpha_{21} \overline{A_{1}} A_{2} U_{1} e^{i \Omega_{1} t}+\alpha_{22} A_{1}^{2} U_{2} e^{i \Omega_{2} t}\right)+\text { c.c. }+ \text { NRT, }
$$

$$
g_{2}^{2}=i\left(\alpha_{21} \bar{A}_{1} A_{2} W_{1} e^{i \Omega_{1} t}+\alpha_{22} A_{1}^{2} W_{2} e^{i \Omega_{2} t}\right)+\text { c.c. }+ \text { NRT },
$$

$$
G_{2}^{ \pm}=0, \quad L_{2}^{ \pm}=0,
$$$$
H_{2}^{1}=\left(i \alpha_{21} F_{1}+\overline{F_{1}^{\prime}} W_{2}+F_{2}^{\prime} \overline{W_{1}}-\overline{F_{1}} U_{2 r}-F_{2} \overline{U_{1}}\right)
$$$$
\times \bar{A}_{1} A_{2} e^{i \Omega_{1}{ }^{t}}+\left(i \alpha_{22} F_{2}+F_{1}^{\prime} W_{1}-F_{1} U_{1 r}\right)
$$$$
\times A_{1}^{\hat{2}} e^{i \Omega_{2} t}+\text { c.c. }+ \text { NRT, }
$$

$$
\begin{aligned}
H_{2}^{2}= & \left(\bar{U}_{1} U_{2}+\bar{W}_{1} W_{2}-\bar{F}_{1} Q_{2 r}-F_{2} \bar{Q}_{1 r}-\bar{F}_{1}^{\prime} F_{2}^{\prime}\right. \\
& \left.+2 \bar{F}_{1} F_{2}\right) \bar{A}_{1} A_{2} e^{i \Omega_{1} t}+\left[\left(U_{1}^{2}+W_{1}^{2}\right) / 2-F_{1} Q_{1 r}\right. \\
& \left.-F_{1}^{\prime 2} / 2+F_{1}^{2}\right] A_{1}^{2} e^{i \Omega_{2} t}+\text { c.c. }+ \text { NRT },
\end{aligned}
$$

$$
\begin{aligned}
2 M_{2}= & \int_{-\Lambda_{0}}^{\Lambda_{0}}\left(2 \bar{A}_{1} A_{2} \bar{F}_{1} F_{2} e^{i \Omega_{1} t}+A_{1}^{2} F_{1}^{2} e^{i \Omega_{2} t}\right) d z+\text { c.c. } \\
& +\mathrm{NRT},
\end{aligned}
$$

$$
g_{3}^{1}=i l \sum_{k=1.2} \alpha_{3 k} A_{k} U_{k} e^{i \Omega_{k} t}+\mathrm{c} . \mathrm{c} .
$$$$
g_{3}^{2}=i / \sum_{k=1}^{2} \alpha_{3 k} A_{k} W_{k} e^{i \Omega_{k} t}+\text { c.c... }
$$

$$
G_{3}^{ \pm}=\mp /\left(\sum_{k=1,2} A_{k} W_{k z}\left(r \pm \Lambda_{0}\right) e^{i \Omega_{k^{t}}}+\text { c.c. }\right) \text {. }
$$

$$
\begin{aligned}
& L_{3}^{ \pm}=\mp l\left(\sum_{k=1,2} A_{k} F_{k}^{\prime}\left( \pm \Lambda_{0}\right) e^{\left.i \Omega_{k^{t}}+\text { c.c. }\right),}\right. \\
& H_{3}^{ \pm}=i l\left(\sum_{k=1,2} \alpha_{3 k^{2}} A_{k} F_{k} e^{i \Omega_{k^{t}}+\text { c.c. }}\right), H_{3}^{2}=0, \quad M_{3}=0,
\end{aligned}
$$

where we have taken into account that $\Omega_{2}=2 \Omega_{1}$ and NRT stands for nonresonant terms, depending on the fast time variable as $\exp (i \Omega t)$, with $\Omega \neq \pm \Omega_{1}, \pm \Omega_{2}$. 


\section{APPENDIX B: LINEAR STABILITY OF THE STEADY STATES OF THE NORMAL FORMS}

The linear stability of a steady state $\left(B_{1}^{s}, B_{2}^{s}\right)$ is analyzed as usual, by first setting $B_{k}-B_{k}^{s}=B_{k}^{1} \exp (\lambda \tau)+B_{k}^{2} \exp (\lambda \tau)$, for $k=1$ and 2 , in (46)-(47) and (6I)-(62), linearizing, and imposing the resulting linear problem to possess nontrivial solutions. Then we obtain the following characteristic equation (the same for both normal forms):

$$
\lambda^{4}+a_{1} \lambda^{3}+a_{2} \lambda^{2}+a_{3} \lambda+a_{4}=0,
$$

where

$$
\begin{aligned}
a_{1}= & 2(1+a), \\
a_{2}= & 4\left|B_{1}^{s}\right|^{2}-\left|B_{2}^{s}\right|^{2}+(1+4 a)+a^{2}+\widetilde{\omega}^{2}+4(\widetilde{\omega}+\widetilde{l})^{2}, \\
a_{3}= & 4(a+1)\left|B_{1}^{s}\right|^{2}-2\left|B_{2}^{s}\right|^{2}+8 a(\tilde{\omega}+\tilde{l})^{2}+2 \widetilde{\omega}^{2} \\
& +2 a(1+a) . \\
a_{4}= & 4\left|B_{1}^{s}\right|^{4}+4\left(a-2 \widetilde{\omega}^{2}-2 \widetilde{\omega}\right)\left|B_{1}^{s}\right|^{2}+\left[1+4\left(\widetilde{\omega}+\widetilde{l}^{2}\right]\right. \\
& \times\left(a^{2}+\widetilde{\omega}^{2}-\left|B_{2}^{s}\right|^{2}\right) .
\end{aligned}
$$

Equation (B1) has the solution $\lambda=0$ if and only if

$$
a_{4}=0 \text {. }
$$

and it exhibits a pair of purely imaginary. conjugate solutions (in fact, $\lambda= \pm \sqrt{-a_{3} / a_{1}}$ ) if and only if $a_{3} / a_{1}>0$ and

$$
a_{1} a_{2} a_{3}-a_{1}^{2} a_{4}-a_{3}^{2}=0 \text {. }
$$

Also, since $a_{1}>0$ the argument principle or the RouthHurwicz criterium readily implies that all roots of (B1) have a strictly negative real part if and only if the left-hand sides of both (B5) and (B6) are strictly positive.

'F. Preiser, D. Schwabe, and A. Sharman. "Steady and oscillatory thermocapillary convection in liquid columus with free cylindrical surface," $\mathrm{J}$. Fluid Mech. 126, 545 (1983).

"Y. Kamotani, S. Ostrach, and M. Vargas, "Oscillatory thermocapillary convection in a simulated floating-zone configuration." J. Cryst. Growth 66, 83 (1984).

${ }^{3} \mathrm{R}$. Brown, "Theory of transport processes in single crystal growth from the melt," AIChE. J. 34, 881 (1988).

"J. C. Melrose, "Model calculations for capillary condensation," AIChE. J. 12, 986 (1966).

${ }^{5}$ J. N. Zasadzinski, J. B. Sweeney, H. T. Davis, and L. E. Scriven, "Finite element calculation of fluid menisci and thin-films in model porous media," J. Colloid Interface Sci. 119. 108 (1987).

${ }^{6}$ T. Y. Chen, J. Tsamopoulos, and R. J. Good, "Capillary bridges between parallel and non-parallel surfaces and their stability," J. Colloid Interface Sci. 151, 19 (1992).
'J. Tsamopoulos, T. Y. Chen, and A. Borkar, "Viscous oscillations of capillary bridges," J. Fluid Mech. 235, 579 (1992).

${ }^{8}$ D. Rivas and J. Meseguer, "One-dinensional self-sinilar solution of the dynamics of axisymmetric slender liquid bridges," J. Fluid Mech. 138. 417 (1984).

${ }^{9}$ Y. Zhang and J. I. D. Alexander, "Sensitivity of liquid bridges subjected to axial residual acceleration," Phys. Fluids A 2, 1966 (1990).

${ }^{10} \mathrm{~J}$. M. Perales and J. Meseguer, "Theoretical and experimental study of vibration of axisymmetric viscous liquid bridges," Phys. Fluids A 4, 1110 (1992).

"F. J. Gareía and A. Castellanos. "One-dimensional models for slender axisynmetric viscous licuid bridges," Phys. Fluids 8, 2837 (1996).

${ }^{12}$ R. M. S. M. Schulkes, "Nonlinear dyoamics of liquids columns: A conparative study," Phys. Fluids A 5, 2121 (1993).

${ }^{13} \mathrm{~A}$. Sanz, "The influence of the outer bath in the dynamics of axisymmetric liquid bridges," J. Fluid Mech. 156. 101 (1985).

${ }^{14} \mathrm{~A}$. Sanz and J. L. Diez. "Non-axisymmetric oscillations of liquid bridges," J. Fluid Mech. 205, 503 (1989).

${ }^{15}$ J. A. Nicolas. "Hydrodynamic stability of high-viscosity cylindrical liquid bridges," Phys. Fluids A 4, 1620 (1992).

${ }^{16} \mathrm{M}$. Higuera, J. A. Nicolás, and J. M. Vega, "Linear oscillations of weakly dissipative axisymmetric liquid bridges," Phys. Fluids A 6, 438 (1994).

${ }^{17} \mathrm{M}$. Hignera and J. A. Nicolas, "Linear non-axisymmetric oscillations of nearly-inviscid liquid bridges," Phys. Fluids 9. 276 (1997).

${ }^{18} \mathrm{~T}$. Y. Chen and J. Tsamopoulos, "Nonlinear dynamics of capillary bridges: Theory," J. Fluid Mech. 255, 373 (1993).

${ }^{19} \mathrm{~J}$. A. Nicolas and J. M. Vega, "Weakly nonlinear oscillations of nearlyinviscid axisymmetric liquid bridges," J. Fluid Mech. 328, 95 (1996).

${ }^{20}$ A. V. Anilkumar, R. N. Grugel, X. F. Shen, C. P. Lee, and T. G. Wang. "Control of thermocapillary convection in a liquid bridge by vibration," J. Appl. Plys, 73, 4165 (1993).

${ }^{21}$ D. J. Mollot. J. Tsamopoulos. T. Y. Chen, and N. Ashgriz, "Nonlinear dyoamics of capillary bridges: Experiments," J. Fluid Mech. 255. 411 (1993).

${ }^{22}$ J. A. Nicolas, D. Rivas, and J. M. Vega. "The interaction of thermocapillary convection and low-frecuency vibration in nearly-inviscid liquid bridges," Z. Angew. Math. Phys. 48, 389 (1997).

${ }^{23}$ J. A. Nicolas. D. Rivas, and J. M. Vega, "On the steady streaming flow due to high-frequency vibration in nearly-ioviscid liquid bridges." J. Fluid Mech. 354, 147 (1998).

${ }^{24}$ M. Higuera, J. A. Nicolás, and J. M. Vega. "The normal form that applies to the weakly nonlinear description of counter-rotating surface waves in capillary bridges and in related geometries," preprint 1997.

${ }^{23}$ Z. C. Feng and L. G. Leal, "Bifurcation and chaos in shape and volume oscillations of a periodically driven bubble with two-to-one internal resonance." J. Fluid Mech. 266. 209 (1994).

${ }^{26} \mathrm{~F}$. Ursell, "Edge waves on a sloping beach," Proc. R. Soc. London, Ser. A 214, 79 (1952)

${ }^{27} \mathrm{C}$. C. Mei and L. F. Liu, "The damping of surface gravity waves in a bounded liquid," J. Fluid Mech. 59, 239 (1973).

${ }^{28} \mathrm{H}$. Schlichting Boundary Layer Theory (McGraw-Hill, New York, 1968).

${ }^{29}$ A. Wolf, J. B. Swift, H. L. Swimney, and J. A. Vastano, "Determining Lyapunov exponents from a time series," Physica D 16. 285 (1985).

${ }^{30} \mathrm{H}$. B. Keller, Lectures on Numerical Methods in Bifurcation Problems, Tata Institute of Fundamental Research. Bombay (Springer-Verlag, Berlin, 1987).

${ }^{31}$ R. C. Hilborn. Chaos and Nonhnear Dynamics (Oxford University Press. Oxford. 1994). 